\title{
Tandemly repeated DNA families in the mouse genome
}

\author{
Aleksey S Komissarov ${ }^{1}$, Ekaterina V Gavrilova ${ }^{1,2,3}$, Sergey Ju Demin ${ }^{1}$, Alexander M Ishov ${ }^{3}$ and Olga I Podgornaya $a^{1,2^{*}}$
}

\begin{abstract}
Background: Functional and morphological studies of tandem DNA repeats, that combine high portion of most genomes, are mostly limited due to the incomplete characterization of these genome elements. We report here a genome wide analysis of the large tandem repeats (TR) found in the mouse genome assemblies.

Results: Using a bioinformatics approach, we identified large TR with array size more than $3 \mathrm{~kb}$ in two mouse whole genome shotgun (WGS) assemblies. Large TR were classified based on sequence similarity, chromosome position, monomer length, array variability, and GC content; we identified four superfamilies, eight families, and 62 subfamilies - including 60 not previously described. 1) The superfamily of centromeric minor satellite is only found in the unassembled part of the reference genome. 2) The pericentromeric major satellite is the most abundant superfamily and reveals high order repeat structure. 3) Transposable elements related superfamily contains two families. 4) The superfamily of heterogeneous tandem repeats includes four families. One family is found only in the WGS, while two families represent tandem repeats with either single or multi locus location. Despite multi locus location, TRPC-21A-MM is placed into a separated family due to its abundance, strictly pericentromeric location, and resemblance to big human satellites.

To confirm our data, we next performed in situ hybridization with three repeats from distinct families. TRPC-21AMM probe hybridized to chromosomes 3 and 17, multi locus TR-22A-MM probe hybridized to ten chromosomes, and single locus TR-54B-MM probe hybridized with the long loops that emerge from chromosome ends. In addition to in silico predicted several extra-chromosomes were positive for TR by in situ analysis, potentially indicating inaccurate genome assembly of the heterochromatic genome regions.

Conclusions: Chromosome-specific TR had been predicted for mouse but no reliable cytogenetic probes were available before. We report new analysis that identified in silico and confirmed in situ 3/17 chromosome-specific probe TRPC-21-MM. Thus, the new classification had proven to be useful tool for continuation of genome study, while annotated TR can be the valuable source of cytogenetic probes for chromosome recognition.
\end{abstract}

\section{Background}

Tandemly repeated DNA represents a significant portion of the mouse genome and include centromere and pericentromere regions. Although historically referred to as "junk DNA", Tandem Repeats (TR) appear to provide unique structural and functional characteristics due to their tandem organization. Tandemly repeated DNA contains multiple copies of a repeat unit (or monomer) arranged in a head to tail fashion. Centromeres from fission yeast to humans contain TR, and pericentromeric

\footnotetext{
* Correspondence: opodg@yahoo.com

${ }^{1}$ Institute of Cytology RAS, 4 Tikhoretsky avenue, 194064, St. Petersburg, Russia

Full list of author information is available at the end of the article
}

regions enriched in TR appearing to be critically important for establishing heterochromatin formation and proper chromosome segregation [1]. Some of these functions appear to involve RNA interference-mediated chromatin modifications [2-4].

TR content is well investigated in the human genome, and it shows a wide range of repeat sizes and organization, ranging from microsatellites of a few base pairs to megasatellites of up to several kilobases. Microsatellites and Variable Number Tandem Repeats (minisatellites or VNTRs) can be highly polymorphic and thus are used as genetic markers $[5,6]$.

The centromeric region of human chromosomes contains alpha satellite DNA (satDNA), the largest TR
Ciomed Central

(c) 2011 Komissarov et al; licensee BioMed Central Ltd. This is an Open Access article distributed under the terms of the Creative Commons Attribution License (http://creativecommons.org/licenses/by/2.0), which permits unrestricted use, distribution, and reproduction in any medium, provided the original work is properly cited. 
family in the human genome. This family has been extensively studied and provides a paradigm for understanding the genomic organization of TR $[7,8]$. These tandem arrays are composed of either diverged monomers, with no higher order repeat structure, or as chromosome-specific Higher-Order Repeat (HOR) units characterized by distinct periodicity and arrangements of an integral number of basic monomers [9]. The HOR structure of human centromeric alpha satellite is important for centromere function [7].

In humans, the pericentromeric regions consist of alpha satDNA arrays that are surrounded by arrays of "classical" satellites (e.g. human satDNA 1-4) [10-13]. These pericentromeric regions have a specific highorder chromatin structure and might be responsible for chromatin spatial organization.

In the house mouse, Mus musculus, centromeric and pericentromeric regions are represented by two highly conserved, tandemly repeated sequences known as minor and major satellites (MiSat and MaSat, respectively, SATMIN and GSAT_MM in Repbase nomenclature). MiSat are composed of 120-bp AT-rich monomers that occupy 300-600 kb of the terminal region of all mouse telocentric (single-armed) chromosomes; these TR serve as the site of kinetochore formation and spindle microtubule attachment [14-18]. MaSat is more abundant and are combined from 234-bp monomers that resides adjacent to MiSat. MaSat are implicated into heterochromatin formation and sister chromatid cohesion [17,19]. Neither of these satDNA were identified at the centromere of the morphologically distinct acrocentric Y chromosome, which has a very short arm that distinguishes it from the telocentric autosomes and chromosome X [20]. Recently, the centromere of Y chromosome was shown to contain a highly diverged MiSat-like sequence (designated Ymin) with HOR organization previously not described for mouse MiSat arrays [20].

Here we report the analysis of mouse large TR genome organization by a combined bioinformatics and cytological approaches. All large TR found in two mouse whole genome shotgun assemblies (WGS) were classified into four superfamilies, eight families, and 62 subfamilies, including 60 not described yet. The proposed classification is based on array similarity, monomer length, the degree of unit similarity, position on the reference genome chromosome assemblies, and GC content. Three TR were selected for the experimental work due to their abundance in the WGS. All array-based probes recognize chromosomes predicted in silico.

The data reported here represent the overall genome wide assessment of the number, position and organization of large TR in the sequenced mouse genome. Annotated TR could be an important resource for further characterization and overall understanding of the mouse genome.

\section{Results}

Tandemly repeated DNA in mouse whole genome shotgun assemblies

For the initial search of large TR we used two WGS assemblies: Mouse Genome Sequencing Consortium (MGSC) and Celera assemblies [21,22]. The WGS assembly is the entire shotgun sequencing reads assembled into contigs including euchromatic and heterochromatic regions, even when not assembled into gapped contigs or not anchored on chromosomes yet. The regions enriched in TR are mostly not anchored, although TR and in particular satDNA are present in WGS due to their abundance in the genome.

To identify all TR with unit size up to $2 \mathrm{~kb}$ we used TRF (Tandem Repeat Finder [23]). The initial raw TRF output contains data redundancy due to nested repeats and repeats with the same coordinates but different unit sizes. To eliminate this redundancy all nested repeats with array length less than the parent array were removed. In case of same coordinates an array with a longer unit size was removed. Both in MGSC ( 3\%) and Celera ( 5\%) WGS the amount of non-redundant TR is less than the experimentally determined amount of the MaSat alone ( 8\%) [24], indicating that even in WGS data sets TR remain underrepresented (Table 1). Since the mouse genome is enriched with micro- and minisatellites [21], we tried to get rid of them with a filter that excluded any array less than $3 \mathrm{~kb}$. In both WGS collections we found 941 large TR (Table 1), which were further grouped into families due to sequence similarity (Figure 1).

\section{Families and superfamilies}

Each pair of arrays was compared by bl2seq program, and the score value was used as a measure of TR

Table 1 Tandem repeats in mouse WGS assemblies

\begin{tabular}{|c|c|c|c|c|c|c|}
\hline Assembly & GPID & Size (bp) & Contigs & $\begin{array}{l}\text { TR } \\
\text { (all) }\end{array}$ & $\begin{array}{l}\text { \% of } \\
\text { assembly }\end{array}$ & $\begin{array}{l}\text { TR } \\
(>3 \mathrm{~kb})\end{array}$ \\
\hline MGSC WGS & 13183 & $2,477,633,597$ & 224,713 & 849,466 & $2.9 \%$ & 157 \\
\hline Celera WGS & 11785 & $3,003,109,157$ & 837,963 & $1,084,552$ & $5.0 \%$ & 784 \\
\hline Total WGS & & $5,480,742,754$ & $1,062,676$ & $1,934,018$ & $3.8 \%$ & 941 \\
\hline
\end{tabular}

GPID - NCBI Genome Project ID. TR (all) - total amount found in assembly; MGSC - the mouse genome sequencing consortium. 


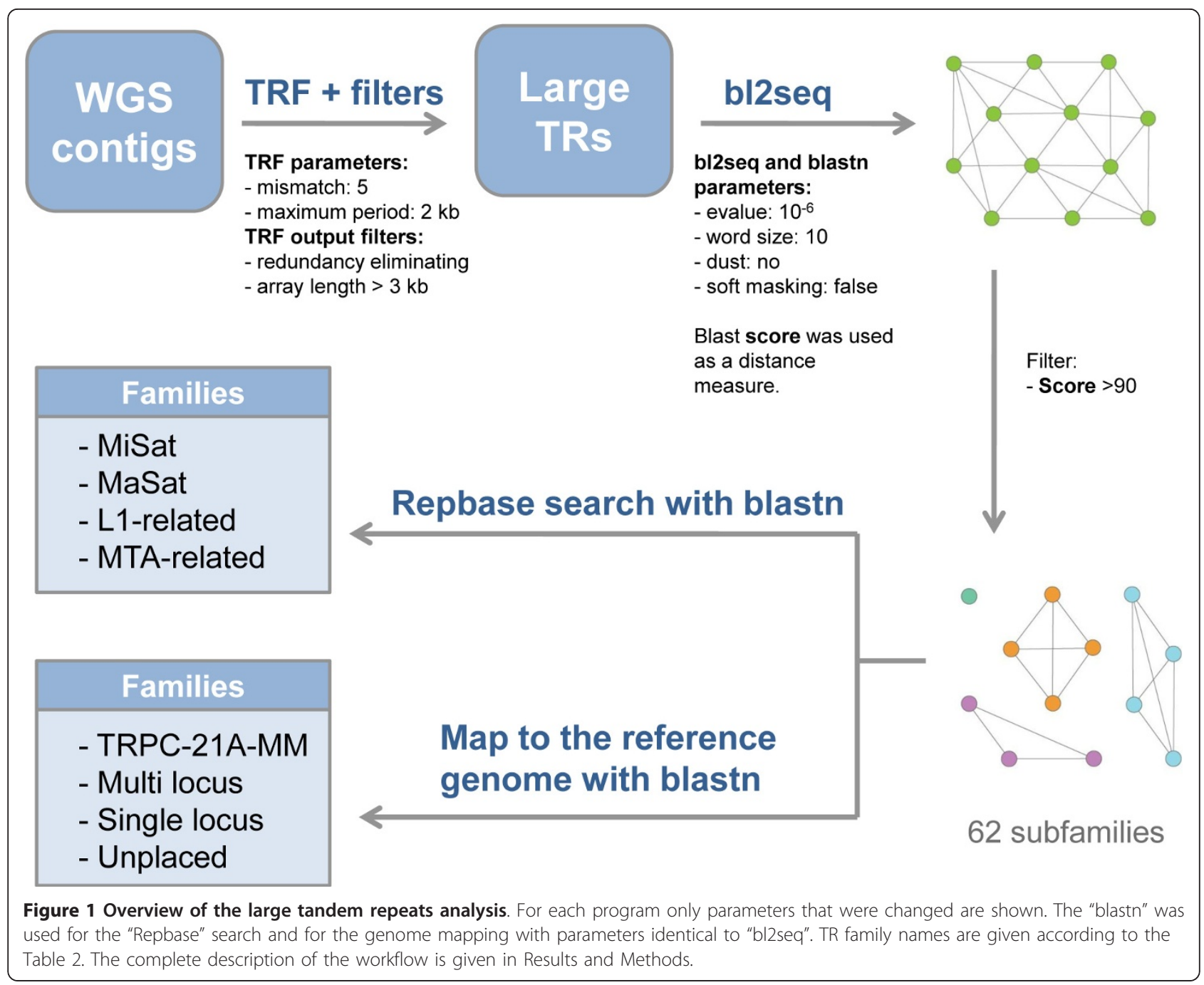

sequence similarity. Two tandem repeats were placed in the same subfamily if they had a bl2seq match with score greater than 90 . This subdivided the TR into 62 subfamilies. We used the Blast search versus rodent Repbase repeat collection to check the similarity with known mouse repeats. This search determined only two known mouse satDNA: 715 arrays $(\sim 76 \%)$ represent pericentromeric MaSat and $21(\sim 2 \%)$ represent centromeric MiSat family (Table 2). The rest of the TR families are not present in Repbase; therefore, they were

Table 2 Mouse large tandem repeats classification

\begin{tabular}{|c|c|c|c|c|c|c|}
\hline Superfamily & $\mathrm{N}$ & Family & Genome Position & Arrays & $\%$ of TR & Subfamilies \\
\hline A. Centromeric & 1 & MiSat & Cen* & 21 & 2.2 & 1 \\
\hline B. Pericentromeric & 2 & MaSat & periCen* & 715 & 76.0 & 1 \\
\hline \multirow[t]{4}{*}{ C. Heterogeneous } & 3 & TRPC-21A-MM & periCen & 50 & 5.3 & 1 \\
\hline & 4 & Multi locus & Any & 57 & 6.0 & 20 \\
\hline & 5 & Single locus & Any & 56 & 6.0 & 29 \\
\hline & 6 & Unplaced & Absent & 11 & 1.2 & 8 \\
\hline \multirow[t]{2}{*}{ D. TE-related } & 7 & MTA related & Any & 15 & 1.6 & 1 \\
\hline & 8 & L1_MM related & Any & 16 & 1.7 & 1 \\
\hline
\end{tabular}

Eight families of the TR found in WGS were combined in four superfamilies (A-D). Families are formed according to sequence similarity and/or position in the reference genome. Arrays - the number of TR arrays found in WGS; \% of TR - percent of all TR found in WGS; Subfamilies - number of subfamilies in family. ${ }^{*}$ ) MaSat and MiSat position is determined by in situ data published; (D) - Tandem repeats related to transposable elements (TE); (MTA) - mouse transcript retrotransposon, (L1) - L1_MM. 
named according to their structure and genome position. For two families (C4, C5 in Table 2) the published nomenclature was used: single locus (SL) family for arrays found only once in the reference genome, whereas multi locus (ML) family for arrays found at more than one locus [5]. A subfamily name includes the letters TR (Tandem Repeat), genomic position (if known), minimal unit size in $\mathrm{bp}$, index letter if there is more than one TR with similar unit size (A, B, etc), and suffix MM (Mus Musculus), with the latter present only in the tables and figures.

The characteristic feature of superfamily " $C$ " is the prominent variability of the TR, which could be divided into subfamilies. The most abundant is TRPC-21A, which has a strictly pericentromeric location (Table 2, C3). Multi and single locus families each represent $\sim 6 \%$ of the TR dataset. Some of the TR arrays ( 1\%) from Unplaced family (UnP, Table 2, C6), which have a distinct monomer and relatively long arrays, are not found in the reference genome.

The superfamily " $\mathrm{D}$ " is formed by MTA-related and L1-related families ( $3 \%$ together), which show structural characteristics related to dispersed transposable elements (TE), but are tandemly organized, and have several features quite distinct from the most members of the set (Table 2, D7, D8).

The relationship between families depending on monomer length, the degree of unit similarity and GC content are shown in the graph (Figure 2). The most clear and compact cloud is formed by MaSat arrays though it is not as uniform as might have been expected from the experimental studies [24,25]. MiSat cloud is in proximity to the MaSat but forms a distinct group. In the area of relatively short monomer unit, two defined clouds of TRPC-21A and other multi locus TR are visible. The transposon-related TR form a loose cloud in the region of long monomer units. Arrays from SL and UnP families are scattered throughout the whole plot. It is likely that additional data from oncoming mouse genome resequencing could improve the classification of SL and UnP families.

\section{Chromosome ends}

Even for human, the best assembled mammalian genome, only chromosomes 8 and $\mathrm{X}$ have the higher-order repeat units known to be at the centromeric region $[8,26,27]$. The large regions of classical heterochromatin are poorly assembled [6], and for the mouse genome even less is known. Mouse telocentric chromosomes have extended TR arrays at the ends. That is the reason why these regions are difficult to assemble and chromosomes end abruptly in $3 \mathrm{Mb}$ gaps reserved for centromeric regions.

We identified what kinds of TR are preceding these gaps (Table 3 ). The ends assembly does not allow to find TR on all chromosomes, so we determine the distance from the gap to the first gene (Additional file 1, Table S1). Only two assemblies end up in MaSat arrays: chromosomes 9 and 11. Four assemblies end up in the newly found TRPC-21A (chromosomes 3, 4, 16 and 17).

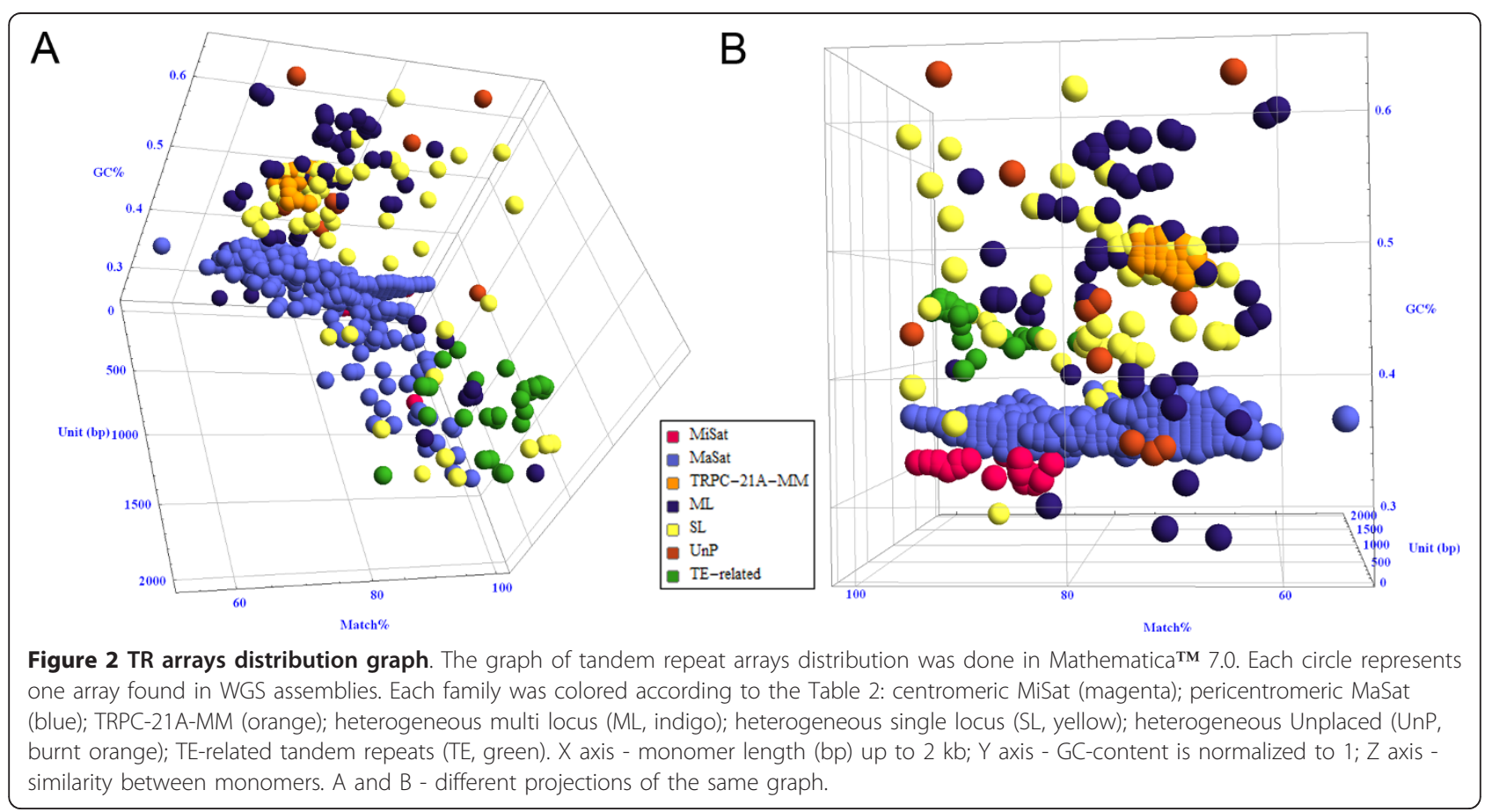


Table 3 TR arrays in the region adjusted to centromeric gap

\begin{tabular}{llll}
\hline Chromosome & TR subfamily & Array length $(\mathbf{k b})$ & Coordinates (bp) \\
\hline 3 & TRPC-21A-MM & 33.6 & $3000001-3033629$ \\
4 & TRPC-21A-MM & 7.0 & $3006469-3013522$ \\
4 & TR-22A-MM & 4.9 & $3104899-3109811$ \\
6 & TR-22A-MM & 9.9 & $3082006-3091879$ \\
9 & MaSat & 38.4 & $3000003-3038419$ \\
11 & MaSat & 3.9 & $3000004-3003872$ \\
16 & TRPC-21A-MM & 9.0 & $3232335-3241336$ \\
17 & TRPC-21A-MM & 32.5 & $3006399-3038945$ \\
17 & TR-27A-MM & 4.6 & $3070530-3075093$ \\
18 & TR-22A-MM & 8.0 & $3112790-3120776$
\end{tabular}

Only TR with the array more than $3 \mathrm{~kb}$ in the distance up to $2 \mathrm{Mb}$ from the centromeric gap is shown. TR - TR name is given according to Tables 4 and 5 . Coordinates - the array position on chromosome.

On chromosomes 4 and 17 the arrays of TR-22A and TR-27A are followed by TRPC-21A. TR-22A arrays are also found at the very ends of chromosomes 6 and 18 . We found out that only eight chromosome ends contain TR arrays and six of them are distinct from the pericentromeric MaSat.

MiSat (minor satellite) and MaSat (major satellite) families The previous experimental data indicated the sequence uniformity of mouse satDNA, i.e. MaSat monomers variability is less than $5 \%$ [25], and $~ 5.6 \%$ variation is found between MiSat monomers [28]. MaSat and MiSat are both AT-rich (64\% and 66\% respectively), and share stretches of sequences with $83 \%$ homology [16]. MiSat arrays were not found in the assembled reference genome. However, Chromosome Unknown (ChrUn) contains MiSat (Additional file 1, Table S2). Centromeric position of MiSat in Table 2 is given according to fluorescent in situ hybridisation (FISH) [29-31]. All the MiSat arrays (the longest array is $\sim 6 \mathrm{~kb}$ ) are AT-rich, with GC content no more than 33\%. Monomer variability of MiSat family is the lowest of all families except TE-related superfamily. In accordance with the data published $[18,20,28,32]$ and low monomer variability MiSat arrays do not have a prominent HOR structure. One third of the arrays have the $120 \mathrm{bp}$ monomer unit reported for MiSat [14,28,32]. The rest has units of 112 bp, $223 \mathrm{bp}, 232 \mathrm{bp}$ and one of the units is $1054 \mathrm{bp}$. The unit difference may be a base for the HOR structure, but the limited number of MiSat arrays found in WGS makes it difficult to draw conclusions on this point right now.

The pericentromeric AT-rich MaSat is formed by 234 bp heterotetramer that consists of four different $58-60 \mathrm{bp}$ monomers with common motif [24]. MaSat is the most abundant family in WGS (Table 2). Very few MaSat arrays found in the WGS exceed $10 \mathrm{~kb}$, with the longest being $23 \mathrm{~kb}$ (Additional file 2, NN 234 and 316). The array of $38 \mathrm{~kb}$ is found at the end of chromosome 9 in the reference genome (Table 3). This feature, the array length, differs from the human genome, where alpha satDNA are assembled in arrays with length $>100 \mathrm{~kb}$ [6]. The MaSat family has GC content no more than 37\% and the mean monomer variability of $30 \%$. The MaSat has two common unit size variants: $35 \%$ of arrays have the experimentally described 58-59 bp monomer [24] and $31 \%$ have the 234 bp classical monomer (Figure 3 ). MaSat arrays with short monomers have the most prominent variability ( $\sim 30 \%$ for 58 bp unit). Arrays with 234 bp monomer show the lowest rate of the variability, with a mean of $15 \%$ (NN 397-617 in Additional file 2). Very few of the arrays have variability about $5 \%$. Thus, bioinformatics approach does not confirm the high degree of MaSat sequence conservation that was concluded from the experimental data [25].

The high rate of the unit variability suggests the existence of a HOR structure in the array. This was checked with a dot-plot similarity analysis where the sequence is self-compared with the fixed 13 bp window (Figure 4). A degree of similarity is indicated by a greyscale where a darker grey represents higher degree of similarity. Therefore, repeated units with high similarity look like diagonal lines, and repeated motifs look like square patterns. We found that about $60 \%$ of MaSat arrays have a HOR structure with a clear "tartan" pattern (Figure 4A). A conservative 234 bp heterotetramer $(58+60+58+58$ bp units) is visible at higher magnification (Figure 4C). Moreover, each unit consists of two less conservative 28 bp and 30 bp subunits (Figure 4D).

TRF output contained MaSat arrays with a unit size of more than 1000 bp (Figure 3; Additional file 2, NN 698715). It is likely that MaSat has units even larger than 2 $\mathrm{kb}$, which are not detected by the TRF search that was restricted to a maximal unit size of $2 \mathrm{~kb}$. Nevertheless the black and white dot-plot with $51 \mathrm{bp}$ window size demonstrates the overall difference between HORs in different MaSat arrays and confirms the existence of $\sim 2$ kb HOR (Additional file 3, Figure S1A, B).

A prominent difference between MaSat arrays could be expected from dot-plot analysis (Figure 4A). The form of MaSat cloud on Figure 2 also suggests that MaSat is not as uniform as it was previously thought [30]. We suppose that being cloned and assembled each MaSat array might come to the different chromosomes, and then chromosome specificity could be suspected for MaSat previously counted as uniform.

\section{TRPC-21A-MM family}

The second largest family in WGS is TRPC-21A (Heterogeneous TR, family C3, Table 2). It is more GC-rich in comparison to MiSat and MaSat, but its monomer 


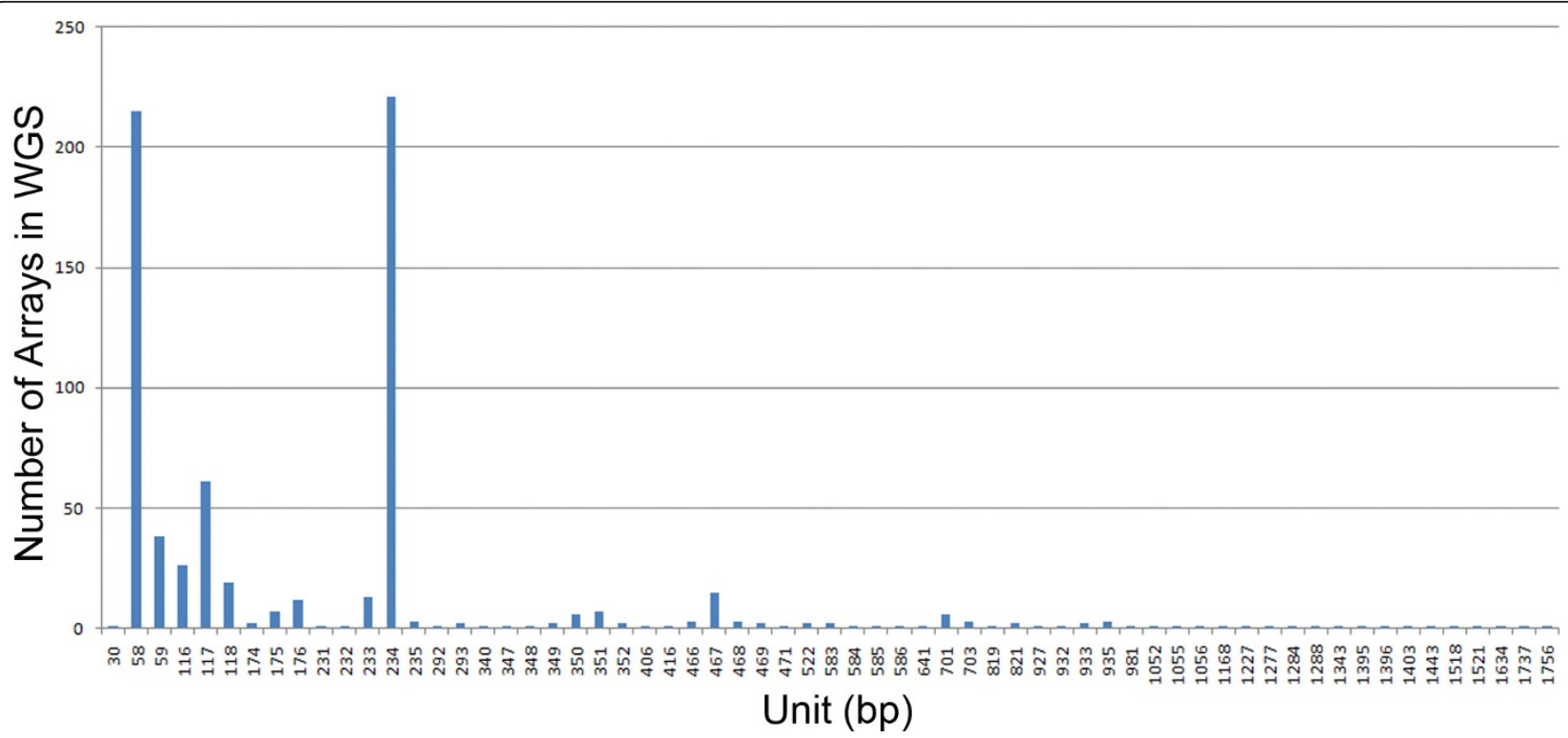

Figure 3 MaSat unit length distribution. X axis - unit length (bp); Y axis - number of the arrays with correspondent unit. The detailed data are shown in Additional file 2. Two main peaks represent 58-59 bp and 234 bp units; presence of larger units can be interpreted as the HOR structure for MaSat.

variability is nearly the same (Table 4). In four cases, when it was found in the assembled genome, it is localized to the very end of centromeric gap (Table 3). Only on chromosome 7 it is placed in the internal band (7D1, Table 4). Moreover, TRPC-21A arrays are found in ChrUn which contains mostly pericentromeric regions (indicated by PC suffix in TRPC-21A name).

All TRPC-21A arrays were divided into ten groups according to the similarity to the specific locus in the reference genome (Table 4). The longest array of $\sim 30$ kb (N35 in Additional file 1, Table S3) probably belongs to chromosome 17 due to the high sequence and length similarity with the array at the end of this chromosome (Table 3). Most arrays show similarity with the band 3A that has the large TRPC-21A field at the end of chromosome (Tables 3 and 4).

Arrays of TRPC-21A are organized by multiplication of the basic 21 bp unit, although TRPC-21A arrays are more homogeneous than MaSat arrays (Figure 5). All TRPC-21A arrays have a HOR structure on dot-plot. In this case even 60-mer units appeared (Figure 5A). PCR with specific primers on the template of total $M$. musculus DNA gave the ladder for TRPC-21A as well as for MaSat, indicating the characteristic feature of the satDNA, also caused by variable monomers organized in HOR (data not shown).

All the features of TRPC-21A are those of a "big classical" satDNA such as human satellites 1-4 [33]. They are known to be chromosome-specific. For example, the bulk of human satellite 3 (HS3) is located on chromosome 1, but it could be distinguished from HS3 on chromosome 9 [34]. To design a FISH probe for TRPC-21A we selected the array with a high similarity to the band 3A2.

\section{Multi locus, single locus and unplaced families}

The Heterogeneous TR superfamily (Table 2) is classified into families according to their presence (ML, SL) or absence (UnP) in the reference genome (Tables 5, 6, and 7). The most abundant ML subfamily, TR-22A, was found in four loci in the reference genome; three are associated with centromeric gap (Table 3and 4A2, 6A2, $18 \mathrm{~B} 2$ in Table 5) and one is located more distant from the centromeric gap (7A2, Table 5).

ML TR-4A consists of a very short AT-rich unit. About a half of the ML subfamilies is present on the sex chromosomes (Table 5). It could be explained by more accurate assembly of the heterochromatic regions on the sex chromosomes relative to autosomes. On the other hand, it is known that the sex chromosomes have unique DNA repeats [35-37] and ML TR-4A can be one of them.

Despite the minimal sequence similarity, several ML and SL subfamilies have similar GC-content, unit size, and array variability, forming three visually distinct groups (clouds) on the graph: GC-rich, AT-rich, and GC-neutral (Figure 2).

TR-22A subfamily is the core of GC-rich cloud in the area of $55-60 \%$ GC, while TR-6A, TR-57A, TR-16A and TR-31B are closely adjoined. At least one subfamily 


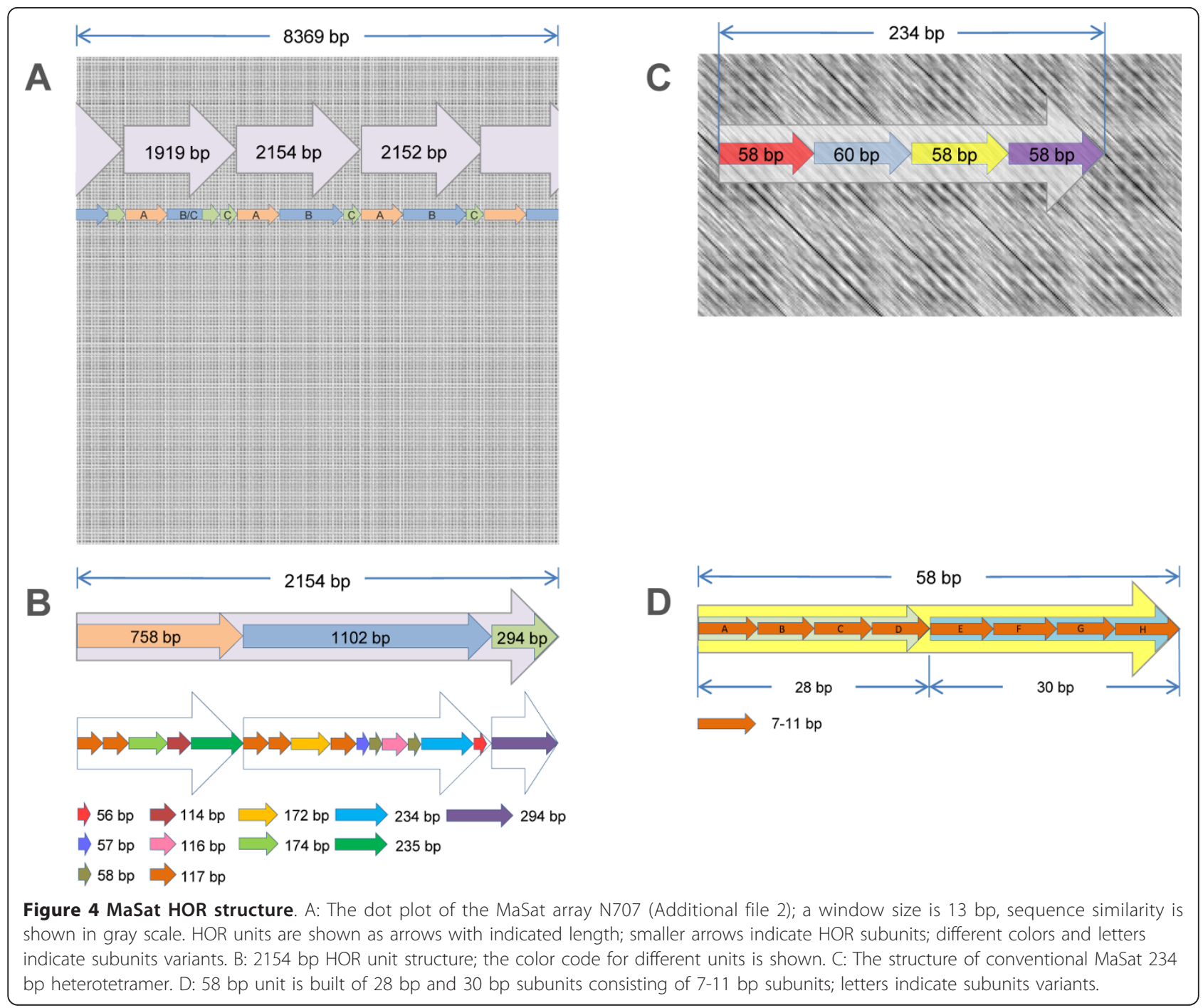

\section{Table 4 TRPC-21A-MM family}

\begin{tabular}{|c|c|c|c|c|c|c|}
\hline $\bar{N}$ & $\begin{array}{l}\text { Unit } \\
\text { (bp) }\end{array}$ & Chromo Bands & Arrays & $\begin{array}{l}\text { GC } \\
\%\end{array}$ & $\begin{array}{l}\text { Length } \\
\text { (bp) }\end{array}$ & $\begin{array}{l}\text { Var } \\
\%\end{array}$ \\
\hline 1 & $42^{*}$ & $3 \mathrm{~A} 2$ & 1 & 49.3 & 4739 & 27 \\
\hline 2 & 21 & $3 \mathrm{~A} 2,4 \mathrm{~A} 2$ & 7 & 50.1 & 5288 & 29 \\
\hline 3 & 63 & $3 \mathrm{~A} 2,17 \mathrm{~A} 2$ & 2 & 48.6 & 4417 & 29 \\
\hline 4 & 42 & $16 \mathrm{~A} 2,17 \mathrm{~A} 2$ & 2 & 48.2 & 29884 & 31 \\
\hline 5 & 21 & 7D1,16A2,17A2 & 6 & 50.1 & 4956 & 29 \\
\hline 6 & 21 & $3 \mathrm{~A} 2,4 \mathrm{~A} 2,17 \mathrm{~A} 2$ & 7 & 48.9 & 7698 & 30 \\
\hline 7 & 21 & $3 \mathrm{~A} 2,16 \mathrm{~A} 2,17 \mathrm{~A} 2$ & 5 & 48.7 & 17198 & 31 \\
\hline 8 & 21 & $3 \mathrm{~A} 2,7 \mathrm{D} 1,16 \mathrm{~A} 2,17 \mathrm{~A} 2$ & 18 & 49.5 & 15684 & 29 \\
\hline 9 & 209 & $3 \mathrm{~A} 2,4 \mathrm{~A} 2,16 \mathrm{~A} 2,17 \mathrm{~A} 2$ & 1 & 48.4 & 7481 & 29 \\
\hline 10 & 21 & $3 \mathrm{~A} 2,4 \mathrm{~A} 2,7 \mathrm{D} 1,16 \mathrm{~A} 2,17 \mathrm{~A} 2$ & 1 & 49.4 & 8021 & 29 \\
\hline
\end{tabular}

Arrays of the TRPC-21A-MM family (Heterogeneous superfamily, Table 2, C3). $\mathrm{N}$ - row index; Unit (bp) - minimal unit length; Chromo Bands - chromosomal positions in the reference genome; Arrays - number of arrays in WGS with similarity to those chromo bands; GC\% - mean array GC content; Length (bp) - maximal array length; Var\% - mean variability between monomers in array. All arrays have HOR and present in ChrUn, so correspondent columns are omitted from this table.

* this sequence was used for the FISH probes design. from SL, TR-31D, also belongs to this group (Additional file 1, Table S3).

The core of AT-rich cloud in the area of $40-45 \%$ GC is formed by subfamilies from SL (TR-17A, TR-38A, TR-39A and other). However, several ML, such as TR81A, also gravitate towards this cloud. Several UnP arrays (TR-24B, TR-28A and other) belong to this group as well (Figure 2 Table 7). Two of subfamilies from ATrich cloud (TR-39A, TR-44A) are embedded into the MaSat cloud or gravitate to MaSat (TR-81A, TR-4A).

Several ML subfamilies (TR-31A, TR-58A) and SL subfamilies (TR-54B, TR-29A, TR-24C) arrays gravitate upon TRPC-21A and form cloud with neutral GC-content (45-55\% GC). Most of them have HOR and are present in ChrUn (Tables 5 and 6).

A number of ML arrays (Table 5, NN 19-22) and SL arrays (Table 6, NN 29-34) have a very long monomer of $>1 \mathrm{~kb}$, although the structure of the long SL and ML 


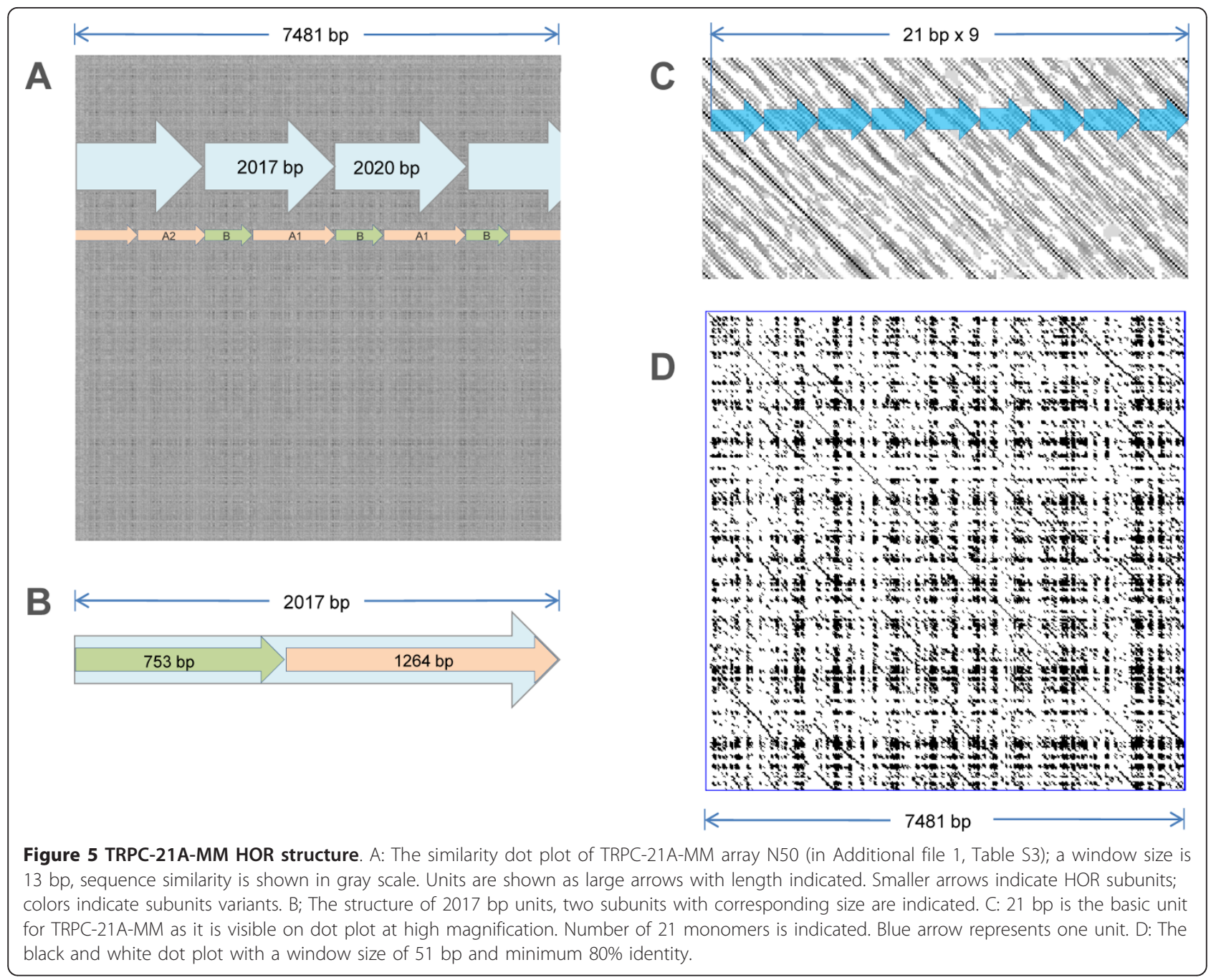

TR does not show extensive similarity with known TEs. However, TR with long units that are classified as ML or SL families could be built on the base of very divergent or unknown TE. The existence of such TEs was predicted in vertebrate genomes [38].

A list of array positions in WGS for the Heterogeneous superfamily is given in the Additional file 1, Table S3.

\section{Transposable elements related tandem repeats}

Two families have structural similarity to transposable elements (TE, superfamily D, Table 2 ). The arrays are formed by the large monomers with a low degree of diversity and similar GC-content in both families (Additional file 1, Table S4).

First family, TR-MTA, is formed by MTA fragments: MTa, MaLR-LTR, Mammalian apparent LTR-retrotransposons in Repbase (Figure 6A). Second family, L1- related family is formed by part of the ORF2 and 3'LTR (Figure 6B).

MTA transposons have structural similarities to endogenous retroviruses, namely ERV3, and are related to THE1 in humans [39]. Endogenous retroviruses by themselves comprise $\sim 10 \%$ of the mouse genome [40]. Over time, most MaLRs have diverged considerably from their consensus sequence, so their number is now estimated at 25-94,000 copies [39]. Preliminary analysis has not yet revealed significant similarities of the putative product of MTA ORF to any protein present in the databanks. The residual part of the ORF is now determined as internal part in MTA Repbase consensus and it is included in TR arrays.

In order to map TE-related arrays to the reference genome two rules were applied. First, a TR hit at a chromosome locus counts as positive only when the alignment length is more than 2850 bp ( $95 \%$ from the 
Table 5 Multi locus family

\begin{tabular}{|c|c|c|c|c|c|c|c|c|c|}
\hline $\bar{N}$ & Subfamily & Unit (bp) & Chromo Bands & Arrays & GC\% & Length & Var\% & ChrUn & HOR \\
\hline 1 & TR-22A-MM & 22 & $\begin{array}{l}4 \mathrm{~A} 2,6 \mathrm{~A} 2 \\
7 \mathrm{~A} 2,18 \mathrm{~B} 2\end{array}$ & 9 & 58.1 & 12896 & 26 & 3 & + \\
\hline 2 & TR-4A-MM & 4 & $\begin{array}{l}1 \mathrm{H} 6,9 \mathrm{~F} 1, \\
9 \mathrm{~F} 3, \mathrm{XA4}, \\
\mathrm{XC2}, \mathrm{XF5}, \\
\text { YA2 }\end{array}$ & 6 & 33.1 & 7704 & 30 & 3 & + \\
\hline 3 & TR-27A-MM & 27 & $14 \mathrm{~B}, 17 \mathrm{~A} 2$ & 4 & 39.6 & 7073 & 27 & 0 & + \\
\hline 4 & TR-31C-MM & 31 & $9 \mathrm{~A} 2^{*}$ & 4 & 49.5 & 13059 & 24 & 0 & + \\
\hline 5 & TR-18A-MM & 18 & $14 \mathrm{~A} 2, \mathrm{XF5}$ & 3 & 55.5 & 5644 & 27 & 1 & + \\
\hline 6 & TR-19B-MM & 19 & $5 \mathrm{G} 1.2,12 \mathrm{D} 1$ & 3 & 47.8 & 5852 & 19 & 0 & + \\
\hline 7 & TR-38C-MM & 38 & $12 \mathrm{~B} 1,13 \mathrm{~A} 3.2$ & 3 & 48.7 & 6797 & 26 & 0 & + \\
\hline 8 & TR-57A-MM & 57 & $\begin{array}{l}5 \mathrm{C} 2,7 \mathrm{~F} 5 \\
8 \mathrm{~A} 1.2,10 \mathrm{D} 3 \\
12 \mathrm{~F} 1,14 \mathrm{~B} \\
16 \mathrm{C} 2,17 \mathrm{~A} 2 \\
\text { XA6 }\end{array}$ & 3 & 52.3 & 5619 & 21 & 0 & + \\
\hline 9 & TR-4B-MM & 4 & $\begin{array}{l}1 \mathrm{H} 6, \mathrm{XA4}, \\
X \mathrm{C} 2\end{array}$ & 2 & 46.2 & 4161 & 36 & 3 & + \\
\hline 10 & TR-6A-MM & 6 & $\begin{array}{l}5 C 2, X C 2 \\
X F 3, X F 5\end{array}$ & 2 & 60.2 & 6649 & 39 & 0 & + \\
\hline 11 & TR-16A-MM & 16 & $\begin{array}{l}6 \mathrm{E} 2,8 \mathrm{~A} 1.2 \\
16 \mathrm{C} 3.2\end{array}$ & 2 & 54.2 & 6765 & 17 & 0 & + \\
\hline 12 & TR-20A-MM & 20 & XA4, XF5 & 2 & 45.5 & 3604 & 31 & 0 & + \\
\hline 13 & TR-31A-MM & 31 & 7D1,8C1 & 2 & 50.6 & 6558 & 20 & 3 & + \\
\hline 14 & TR-31B-MM & 31 & $7 \mathrm{D} 1,14 \mathrm{~A} 2$ & 2 & 53.2 & 4922 & 19 & 1 & + \\
\hline 15 & TR-58A-MM & 58 & $\begin{array}{l}6 \mathrm{~B} 2.2,6 \mathrm{C} 2, \\
6 \mathrm{~F} 3,13 \mathrm{~B} 1\end{array}$ & 2 & 50.5 & 4658 & 34 & 0 & + \\
\hline 16 & TR-1521A-MM & 1521 & $7 F 3, X A 1.2$ & 2 & 44.6 & 3213 & 11 & 1 & + \\
\hline 17 & TR-30A-MM & 30 & $5 B 3,17 \mathrm{~B} 1$ & 1 & 46.3 & 3912 & 37 & 0 & + \\
\hline 18 & TR-81A-MM & 81 & $14 \mathrm{~B}, 17 \mathrm{~A} 2$ & 1 & 40.0 & 3483 & 31 & 0 & + \\
\hline 19 & TR-1164A-MM & 1164 & $X C 2, X D$ & 1 & 48.6 & 3333 & 19 & 0 & - \\
\hline 20 & TR-1595A-MM & 1595 & XA3.2, XA6 & 1 & 38.8 & 3007 & 16 & 0 & - \\
\hline 21 & TR-1149A-MM & 1149 & $X F 1, X F 3$ & 1 & 45.8 & 5463 & 13 & 5 & - \\
\hline 22 & TR-1527A-MM & 1527 & $X C 2, X D$ & 1 & 46.0 & 3120 & 12 & 0 & + \\
\hline
\end{tabular}

Subfamilies ordered by number of arrays in WGS and then by unit length. N - row index; Unit (bp) - minimal unit length; Chromo Bands - chromosomal positions in the reference genome; Arrays - number of arrays of each subfamily found in both WGS; GC\% - mean array GC content; Length (bp) - max array length; Var\% mean variability between monomers in array. ChrUn - number of arrays found in ChrUn; HOR - presence of HOR.

* There are several distinct arrays of TR-31C-MM in 9A2 locus in the reference genome.

original TR array limit of $3 \mathrm{~kb})$. Second, a hit is counted as a single when the distance between two hits is less than 150 bp (5\%). After applying these rules 284 hits with precise positions remained (Additional file 1, Table S5). Most of the loci found for TR-L1 family do not exceed $5 \mathrm{~kb}$. For TR-MTA family we found two loci with array length about $10 \mathrm{~kb}$. All loci were displayed on the banded chromosomes. There is no obvious regularity in TR-MTA family distribution, probably due to the limited amount of the arrays found (Figure 7 , orange). The TR-L1 family is enriched in heterochromatic bands and the concentration on chromosome $\mathrm{X}$ is visible (Figure 7, blue). At the same time no TE-related $\mathrm{TR}$ are found on $\mathrm{Y}$ chromosome. Validation of these findings by FISH is technically challenging, because the LTRs of other retroelements may obscure the results.

\section{Tandem repeat position defined by FISH}

Bioinformatics predictions about the positions of newlyfound TR were checked by in situ experiments. We did not expect to obtain in situ the full correspondence of TR positions found in silico, since the assembly of heterochromatic part of the reference genome is far from being complete. Nevertheless, in silico chromosome locations should be included in the set of the in situ labelled chromosomes. Monomer units from three classes were selected for probe design (see Methods section). All probe sequences with a short description shown in Additional file 1, Table S6. In the reference genome, TRPC-21A has predicted in silico pericentromeric location on four chromosomes (Table 3 and 4) and TRPC-21A arrays were found in ChrUn, which contains mostly pericentromeric regions; therefore, 
Table 6 Single locus family

\begin{tabular}{|c|c|c|c|c|c|c|c|c|c|}
\hline $\mathrm{N}$ & Subfamily & Unit (bp) & Chromo Bands & Arrays & GC\% & Length & Var\% & ChrUn & HOR \\
\hline 1 & TR-17A-MM & 17 & $17 D$ & 5 & 43.2 & 10312 & 33 & 0 & + \\
\hline 2 & TR-54B-MM & 54 & XA1.2 & 5 & 47.9 & 11978 & 31 & 0 & - \\
\hline 3 & TR-29A-MM & 29 & $2 \mathrm{~F} 1$ & 4 & 50.3 & 4175 & 26 & 0 & + \\
\hline 4 & TR-734A-MM & 734 & $x \subset 2$ & 3 & 37.0 & 9507 & 22 & 0 & + \\
\hline 5 & TR-1870A-MM & 1870 & 7D1 & 3 & 44.6 & 5795 & 3 & 8 & - \\
\hline 6 & TR-19A-MM & 19 & $18 \mathrm{~A} 2$ & 2 & 49.7 & 4883 & 32 & 0 & + \\
\hline 7 & TR-34A-MM & 34 & $12 \mathrm{~F} 2$ & 2 & 55.2 & 3354 & 7 & 0 & - \\
\hline 8 & TR-38A-MM & 38 & $8 \mathrm{C} 1$ & 2 & 42.7 & 5426 & 25 & 0 & + \\
\hline 9 & TR-38B-MM & 38 & $8 \mathrm{C} 1$ & 2 & 42.0 & 6113 & 25 & 0 & + \\
\hline 10 & TR-54A-MM & 54 & XA1.2 & 2 & 48.1 & 10744 & 30 & 0 & - \\
\hline 11 & TR-100A-MM & 100 & XA1.2 & 2 & 44.0 & 4364 & 26 & 0 & - \\
\hline 12 & TR-234A-MM & 234 & $3 F 2.2$ & 2 & 59.4 & 6878 & 5 & 0 & - \\
\hline 13 & TR-23A-MM & 23 & $17 \mathrm{~B} 1$ & 1 & 43.4 & 6018 & 13 & 0 & - \\
\hline 14 & TR-24C-MM & 24 & $12 \mathrm{~F} 1$ & 1 & 52.5 & 3298 & 9 & 0 & - \\
\hline 15 & TR-29B-MM & 29 & $2 \mathrm{~F} 1$ & 1 & 50.0 & 15896 & 30 & 0 & + \\
\hline 16 & TR-31D-MM & 31 & $12 \mathrm{~A} 1.2$ & 1 & 55.9 & 5175 & 24 & 1 & + \\
\hline 17 & TR-33A-MM & 33 & $7 \mathrm{~B} 1$ & 1 & 53.4 & 3601 & 17 & 0 & - \\
\hline 18 & TR-39A-MM & 39 & $1 \mathrm{C} 1.2$ & 1 & 39.2 & 3387 & 5 & 0 & - \\
\hline 19 & TR-40A-MM & 40 & $15 \mathrm{~A} 2$ & 1 & 62.6 & 6612 & 21 & 0 & + \\
\hline 20 & TR-44A-MM & 44 & $2 \mathrm{H} 3$ & 1 & 36.3 & 3016 & 9 & 0 & - \\
\hline 21 & TR-48A-MM & 48 & $14 \mathrm{D} 3$ & 1 & 50.7 & 6603 & 28 & 0 & - \\
\hline 22 & TR-56A-MM & 56 & XA1.2 & 1 & 42.9 & 4194 & 22 & 0 & + \\
\hline 23 & TR-84A-MM & 84 & 7E2 & 1 & 48.5 & 3040 & 9 & 0 & - \\
\hline 24 & TR-93A-MM & 93 & $x \subset 2$ & 1 & 51.3 & 3124 & 24 & 3 & - \\
\hline 25 & TR-111A-MM & 111 & 9F4 & 1 & 52.4 & 3347 & 22 & 0 & - \\
\hline 26 & TR-168A-MM & 168 & XA1.2 & 1 & 41.9 & 4046 & 23 & 0 & - \\
\hline 27 & TR-297A-MM & 297 & 17E1.2 & 1 & 56.2 & 3100 & 19 & 0 & - \\
\hline 28 & TR-321A-MM & 321 & $11 \mathrm{E} 2$ & 1 & 42.3 & 3152 & 21 & 0 & + \\
\hline 29 & TR-814A-MM & 814 & $5 \mathrm{~A} 2$ & 1 & 45.5 & 3175 & 3 & 0 & - \\
\hline 30 & TR-1146A-MM & 1146 & $19 B$ & 1 & 47.5 & 3056 & 14 & 0 & - \\
\hline 31 & TR-1284A-MM & 1284 & $10 C 3$ & 1 & 42.3 & 5239 & 14 & 0 & - \\
\hline 32 & TR-1384A-MM & 1384 & XF1 & 1 & 25.8 & 3665 & 8 & 0 & - \\
\hline 33 & TR-1872A-MM & 1872 & $9 \mathrm{~A} 4$ & 1 & 42.5 & 4285 & 17 & 0 & - \\
\hline 34 & TR-1908A-MM & 1908 & $2 \mathrm{~A} 2$ & 1 & 39.8 & 4126 & 14 & 0 & + \\
\hline
\end{tabular}

Subfamilies ordered by number of arrays in WGS and then by unit length. $\mathrm{N}$ - row index; Unit (bp) - minimal unit length; Chromo Bands - chromosomal positions in the reference genome; Arrays - number of arrays of each subfamily found in both WGS; GC\% - mean array GC content; Length (bp) - max array length; Var\% mean variability between monomers in array. ChrUn - number of arrays found in ChrUn; HOR - presence of HOR.

Table 7 Unplaced family

\begin{tabular}{llllllllll}
\hline $\mathbf{N}$ & Subfamily & Unit (bp) & Arrays & GC\% & Length (bp) & Var\% & HOR & ChrUn & WGS Chr \\
\hline 1 & TR-24B-MM & 24 & 3 & 34.4 & 7636 & 28 & + & 2 & 11 \\
2 & TR-24A-MM & 24 & 2 & 45.6 & 3540 & 22 & + & 2 & Un \\
3 & TR-13A-MM & 13 & 1 & 56.0 & 4477 & 15 & + & 0 & 6 \\
4 & TR-27B-MM & 27 & 1 & 63.5 & 3452 & 36 & + & 1 & Un \\
5 & TR-28A-MM & 28 & 1 & 41.2 & 3195 & 23 & + & 1 & Un \\
6 & TR-36A-MM & 36 & 1 & 64.0 & 3003 & 8 & + & 0 & 19 \\
7 & TR-102A-MM & 102 & 1 & 45.7 & 3698 & 31 & - & 0 & $X$ \\
8 & TR-624A-MM & 624 & 1 & 43.3 & 3297 & 2 & - & 0 & Un \\
\hline
\end{tabular}

Subfamilies ordered by number of arrays in WGS and then by unit length. N - row index; Unit (bp) - minimal unit length; Arrays - number of arrays in WGS; GC\% - mean array GC\%; Length (bp) - max array length; Var\% - mean variability between monomers in array. HOR - presence of HOR; ChrUn - arrays found in ChrUn; WGS Chr - chromosome source from WGS contig description. 


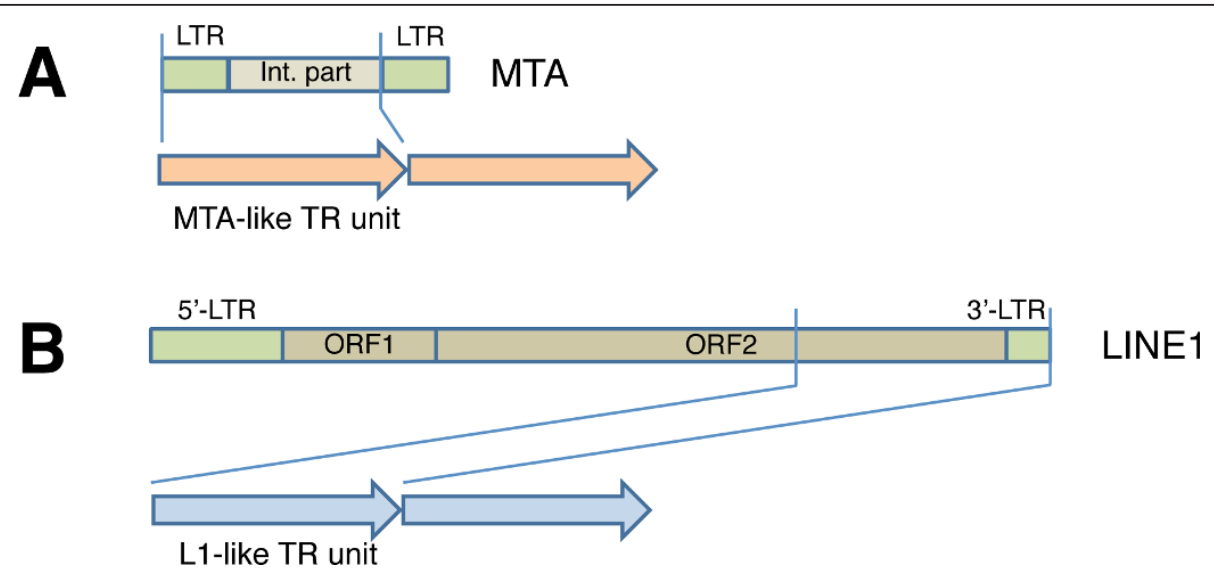

Figure 6 Structure of TE-related tandem repeats. A: The general scheme of MTA related TR family; regions of MTA are denoted, and a fragment of TR unit is marked. B: The general scheme of L1 related TR family; L1 regions are denoted, and a fragment of TR unit is marked.

additional chromosomes have to be labelled in the same region. Single strand dimer labelled from both ends yielded signal on nine chromosomes: $3,5,6,7,8,12,16$, 17 , and Y. The largest signal belongs to chromosome 3 on all chromosome spreads. In each case the label was at the pericentromeric regions except the Y (Figure 8). Four chromosomes predicted as TRPC-21A bearing (Table 4) are in the set of in situ labelled chromosomes.

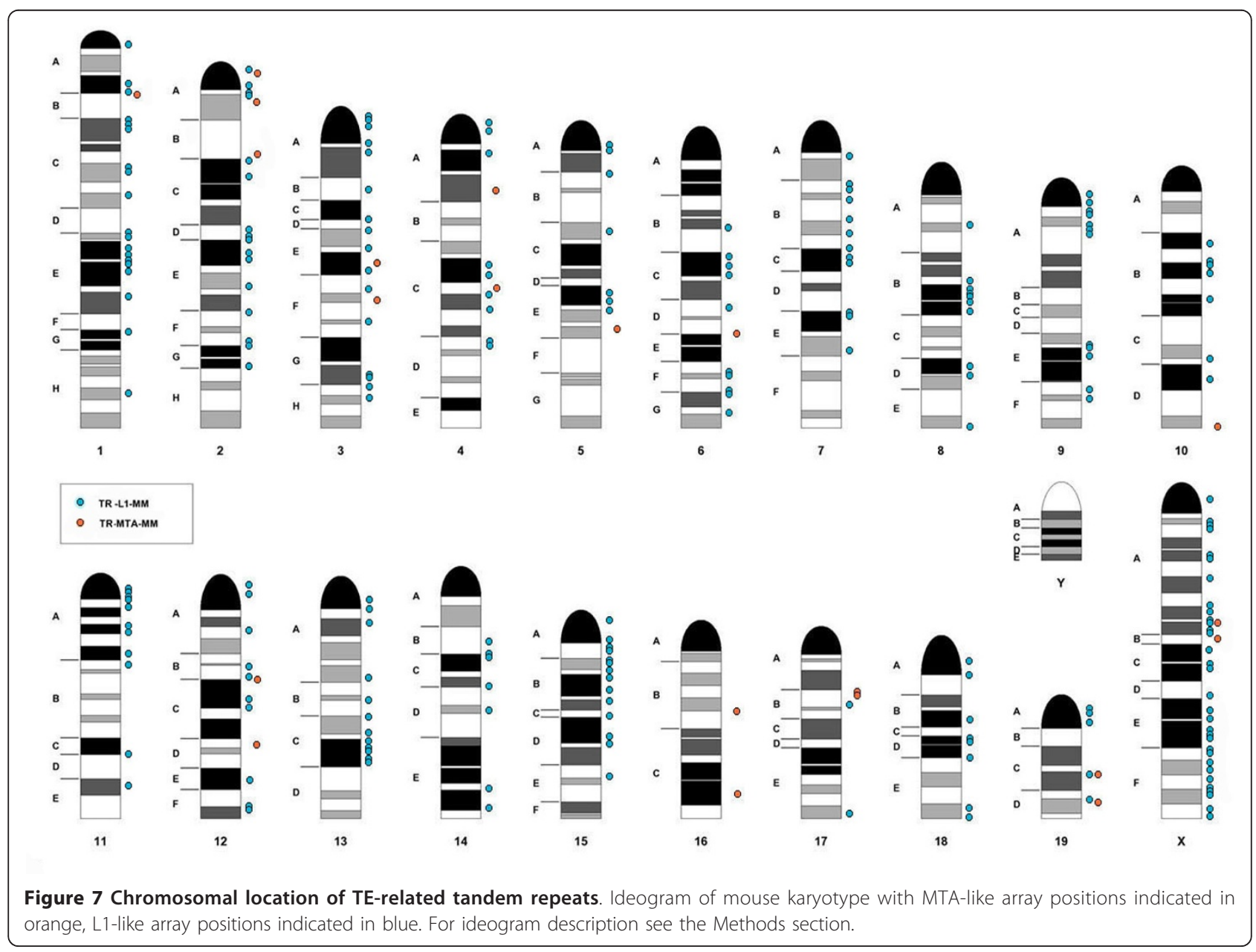



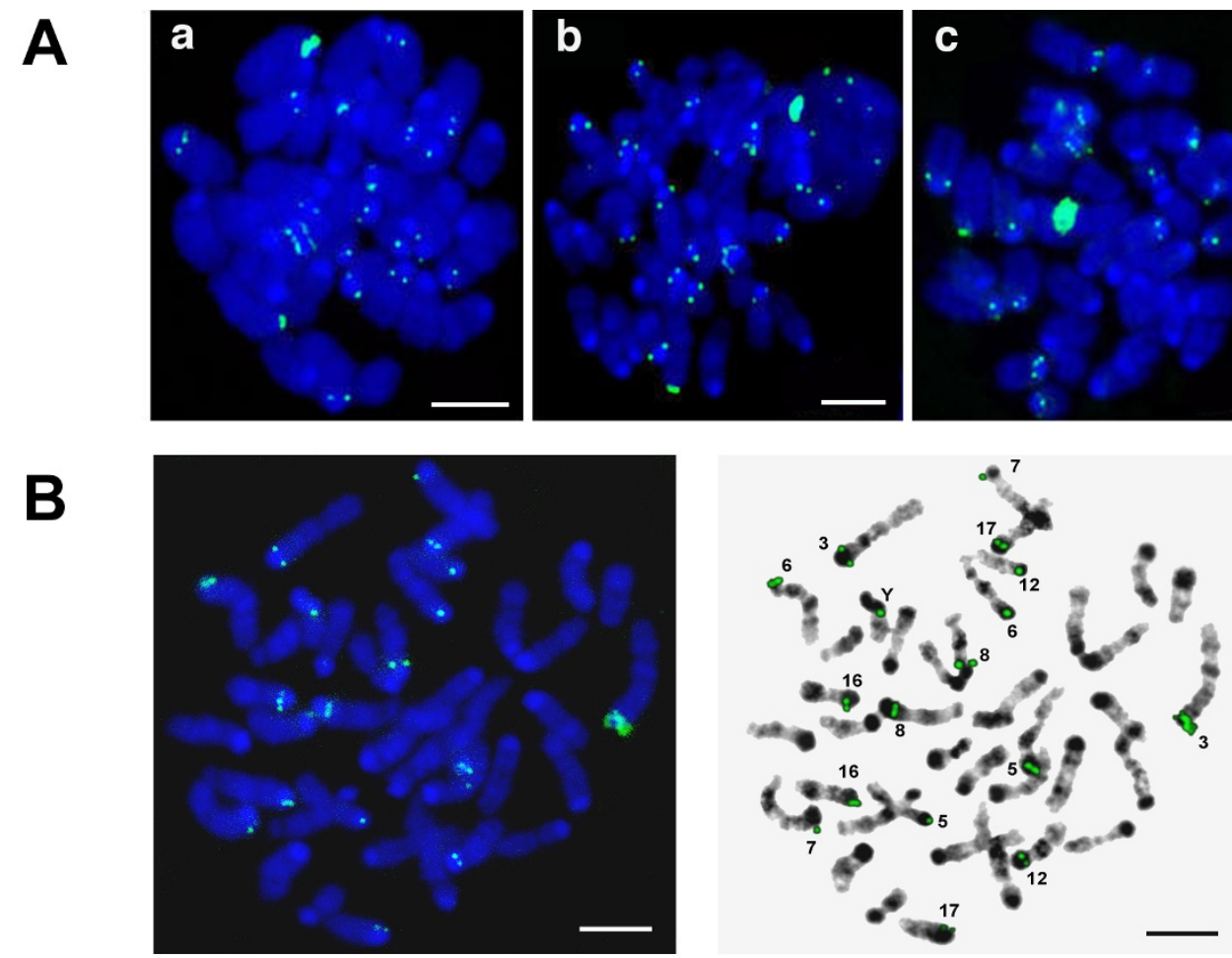

C

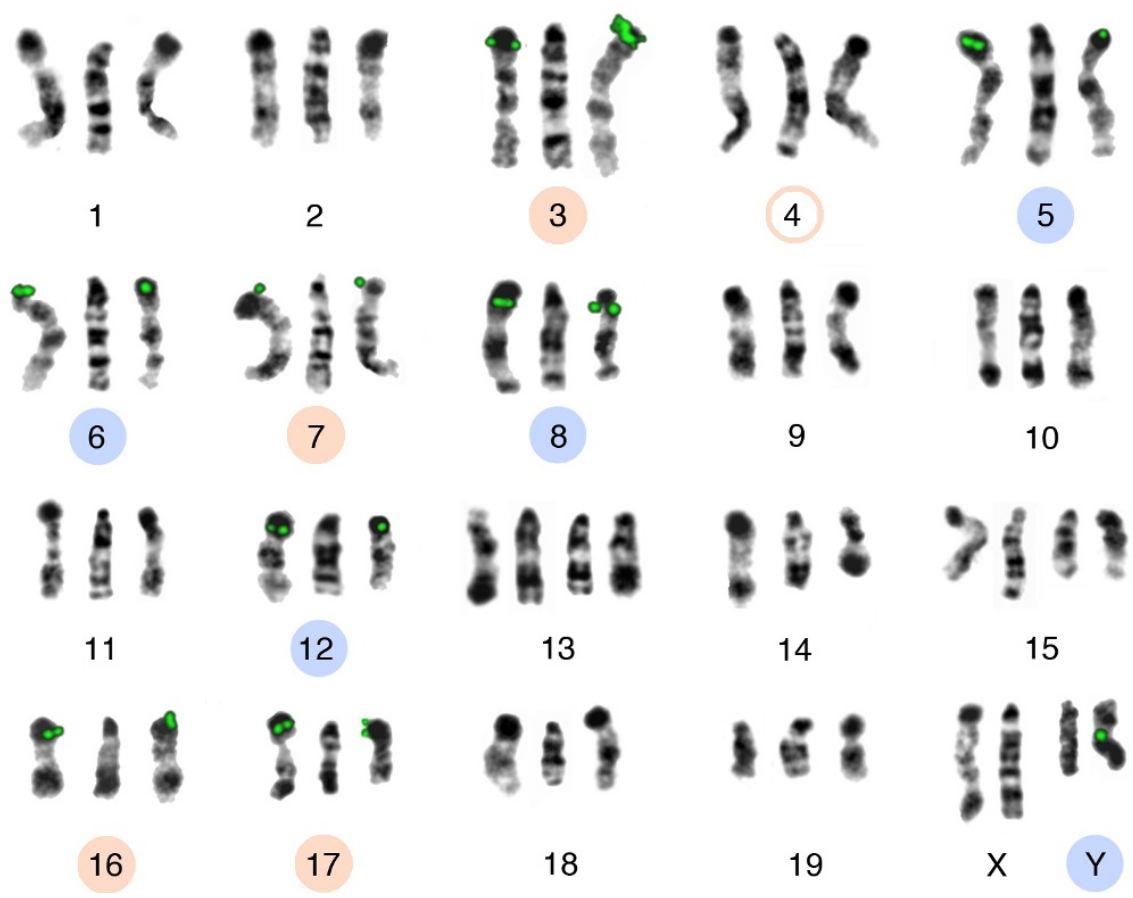

Figure 8 Fluorescent in situ hybridization (FISH) with TRPC-21A-MM short probe. A: bone marrow metaphase plates; B: one of the metaphase sets of chromosomes negative DAPI-banded, numbers of signal bearing chromosomes are indicated. For A and B: DAPI in blue, FISH signal in green; bar - $5 \mu \mathrm{m}$. C: all chromosomes karyotyped. In each group the middle image is G-banded mouse chromosome from atlas [41], the side negative DAPI-banded chromosomes are from the plate shown on B. Nine chromosomes with the label are indicated by circles; four chromosomes, with in situ signal that confirmed in silico prediction, are indicated by orange circle. The assembled chromosome 4 has short TRPC-21A-MM array but does not have signal (indicated by empty orange circle). Chromosome 7 has signal in pericentromeric region instead of predicted in silico $7 \mathrm{D} 1$ band. 
Chromosome 4 has short TRPC-21A array in silico (Table 3) but it lacks any signal, probably due to the wrong assembly. Other discrepancy is the pericentromeric signal seen on chromosome 7, while in silico TRPC-21A mapped to the internal 7D1 band. Instead, Y chromosome has the internal signal, which could be explained by the unique repeats content of the sex chromosomes [20].

The HOR structure of TRPC-21A suggests chromosome-specific variants (Figure 5). The next probe was based on the array fragment from chromosome 3 . The probe is a double stranded $\sim 150$ bp sequence with additional $\sim 20$ bp flanking sequences. Flanks give the possibility to label probe by PCR (Additional file 3, Figure $\mathrm{S} 2$ ). This probe has a strong signal on chromosomes 3 and 17 according to the position of large TRPC-21A arrays at the ends of these chromosomes in the reference genome (Table 3, Figure 9). We suppose that probes designed on the basis of TRPC-21A variants could be specific for other chromosomes.

TR-22A (ML, C4 in Table 2) was chosen for the probe design due to its abundance in the reference genome as well as in ChrUn (Table 5). The monomeric single strand probe labelled from both ends is hybridized to ten chromosomes, four of them predicted as TR-22A bearing (Table 5). In this case the main part of the signal is located at the pericentromeric regions (chromosomes $2,6,7,9,11,17,18$ ), with additional signals located on the arms of chromosomes 2 and 15, and in the subtelomeric region of chromosome 13. In each case signals are located in heterochromatic dark bands (Figure 10). The signal is stronger on L929 chromosome spreads comparing with the signal on normal bone marrow cells (Figure 10Ac). It could be explained by known chromosome polyploidy and rearrangements within heterochromatic regions in L929 cells [30,41]. There is no obvious main signal on any chromosome spread, so the design of chromosome-specific probe on the base of TR-22A could be more complicated and, moreover, the arrays at the ends of chromosomes 4, 6 and 18 in the reference genome do not exceed 10kb (Table 3).

Finally, the SL TR-54B (C5, Table 2) was selected due to the abundance of its arrays at the XA1.2 pericentromeric band. A double strand dimer probe was designed and labelled by PCR. About half of all signals obtained in the late prophase chromosome spreads belong to the long loops emerged from subtelomeric regions of chromosomes during inevitable osmotic shock, which is a necessary step during chromosome-spread isolation $[42,43]$. The signal on the chromosome $X$ is located at the predicted region. However, this signal as well as most of the rest could only be recognized on "fuzzy" chromosomes, when all the DAPI stained material is visible but bands are obscure. In contrast to the reference genome assembly, TR-54B is not a single locus TR, because about fifty signals in total are visible on chromosome spreads (Figure 11). The further mapping of TR-54B using additional probe for the subtelomeric region is required to clarify its exact location.

\section{Discussion}

The computation approaches to the genome-wide TR analysis gradually appear with the genome sequencing advanced [5,6,44-46]. At the chromosomal level TR can be of profound structural as well as evolutionary importance, since genomic regions with a high density of TR, e.g., telomeric, centromeric, and heterochromatic regions, often have specific properties such as alternative DNA structure and packaging [47-49]. At the nuclear level of organization, constitutive heterochromatin may help maintain the proper spatial relationships necessary for the efficient operation of the cell through the stages of mitosis and meiosis. In the interphase nucleus satDNA have one property in common despite their species specificity, namely heterochromatization, which involves RNA interference-mediated chromatin modifications $[2,3,50-54]$. The strand-specific burst in transcription of pericentromeric satellites is required for chromocenters formation in early mouse development. Specific expression dynamics of MaSat repeats, together with their strand-specific control, represent necessary mechanisms during a critical time window in preimplantation development that are of key importance to consolidate the maternal and to set up the paternal heterochromatic state at pericentromeric domains [55]. Such an important and crucial finding is based on the known sequence of the mouse MaSat. Most of the other mouse TR could not be tested in similar experiments being undescribed and unclassified.

\section{Mouse major satellite}

The proportion of MaSat in a total mouse DNA preparation is about $8 \%$, and it is higher than the amount of satDNA found in total DNA preparations from rat and human [24]. MaSat is located near chromosome centromeres [56]. The most wide-spread opinion based on experimental data is the high degree of MiSat and MaSat sequence conservation exists across the telocentric domain of all mouse chromosomes. The earlier publications do not confirm MaSat uniformity. There are data for both short range [57] and long-range periodicity in MaSat [58]. EcoRII digest breaks MaSat into fragments, which form a series of bands on gel electrophoresis (ladder). The DNA in the strongest band was 220-260 bp and the other bands were the multiples of this length. The stronger bands of the minor patterns fall half-way between the bands of the main pattern, and the smallest is 120 to 130 nucleotide pairs long [58]. 


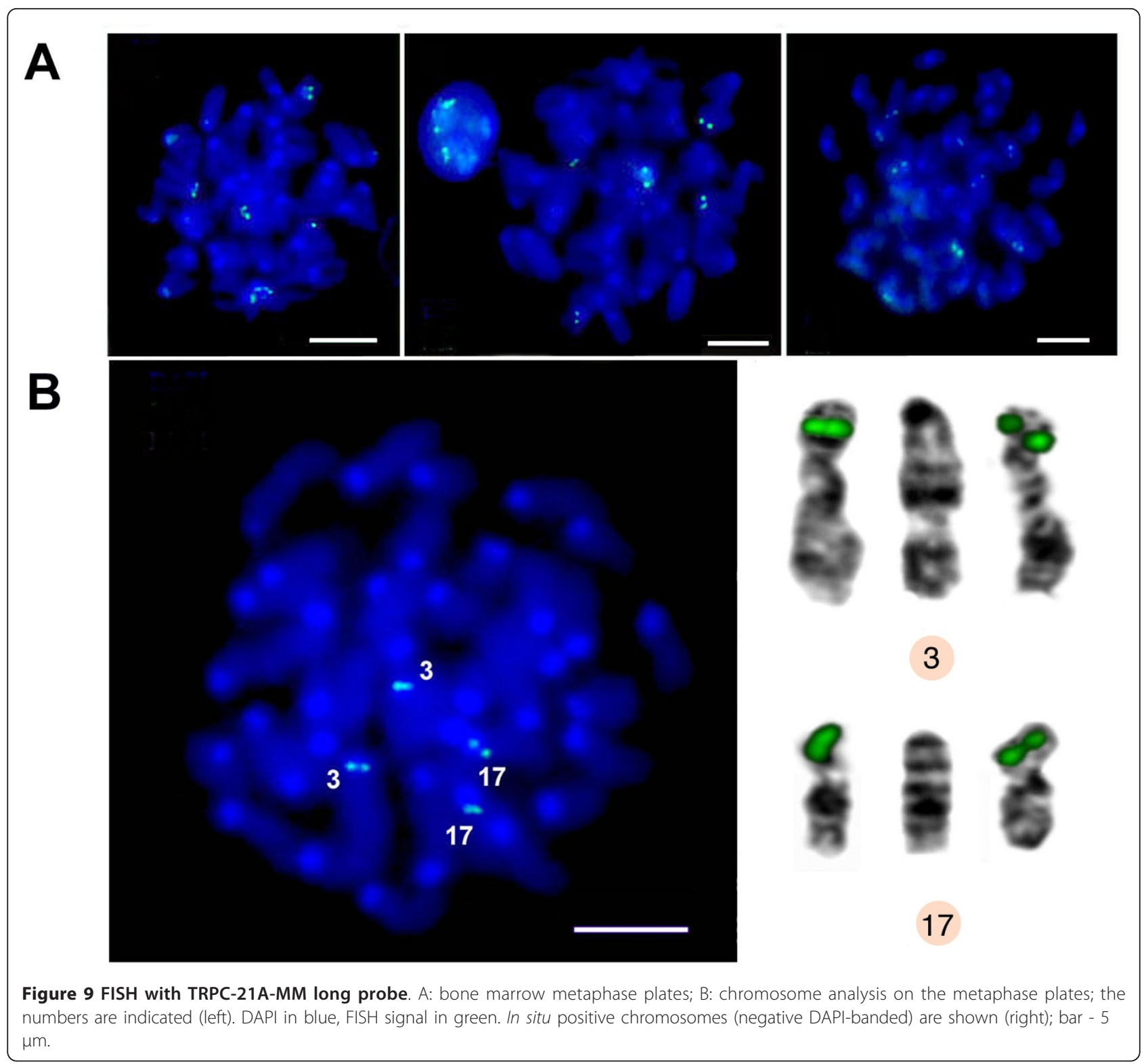

Monomers of the correspondent length are the third in representation among MaSat monomers in the arrays (Figure 3). The sequence is shown to be based on a repeating unit less than $20 \mathrm{bp}$ in length. Four major oligonucleotides were identified, all of which could derive from an original sequence $d\left(G_{5}\right.$ TGA) for the light strand [57]. Short units of the size similar to the reported oligonucleotides could be tracked by MaSat dot-plot analysis (Figure 4D). In contrast to proposed MaSat uniformity based on limited experimental data [25], our results indicate that its monomers variation is quite high. Despite the abundance of MaSat in TRF outputs, the majority of MaSat is unplaced and in all likelihood will be placed in $3 \mathrm{Mb}$ centromeric gaps on each chromosome. We suppose that MaSat arrays could be chromosome-specific and thus may come to different chromosomes during attempts to fill centromeric gap. For this purpose the probes based on different MaSat variants could be designed and checked by FISH.

\section{Mouse minor satellite}

There were previous attempts to find MiSat chromosome-specific variants. MiSat specificity has been shown to chromosome 2 with synthetic oligonucleotide probes and Southern hybridization [59]. Oligonucleotide probes that specifically detect sequence variations were found in some cloned MiSat fragments, and they detected a limited subset of MiSat arrays using pulse-gel electrophoresis with Southern hybridization and PRINS (primed in situ hybridization). Mostly prominent label 

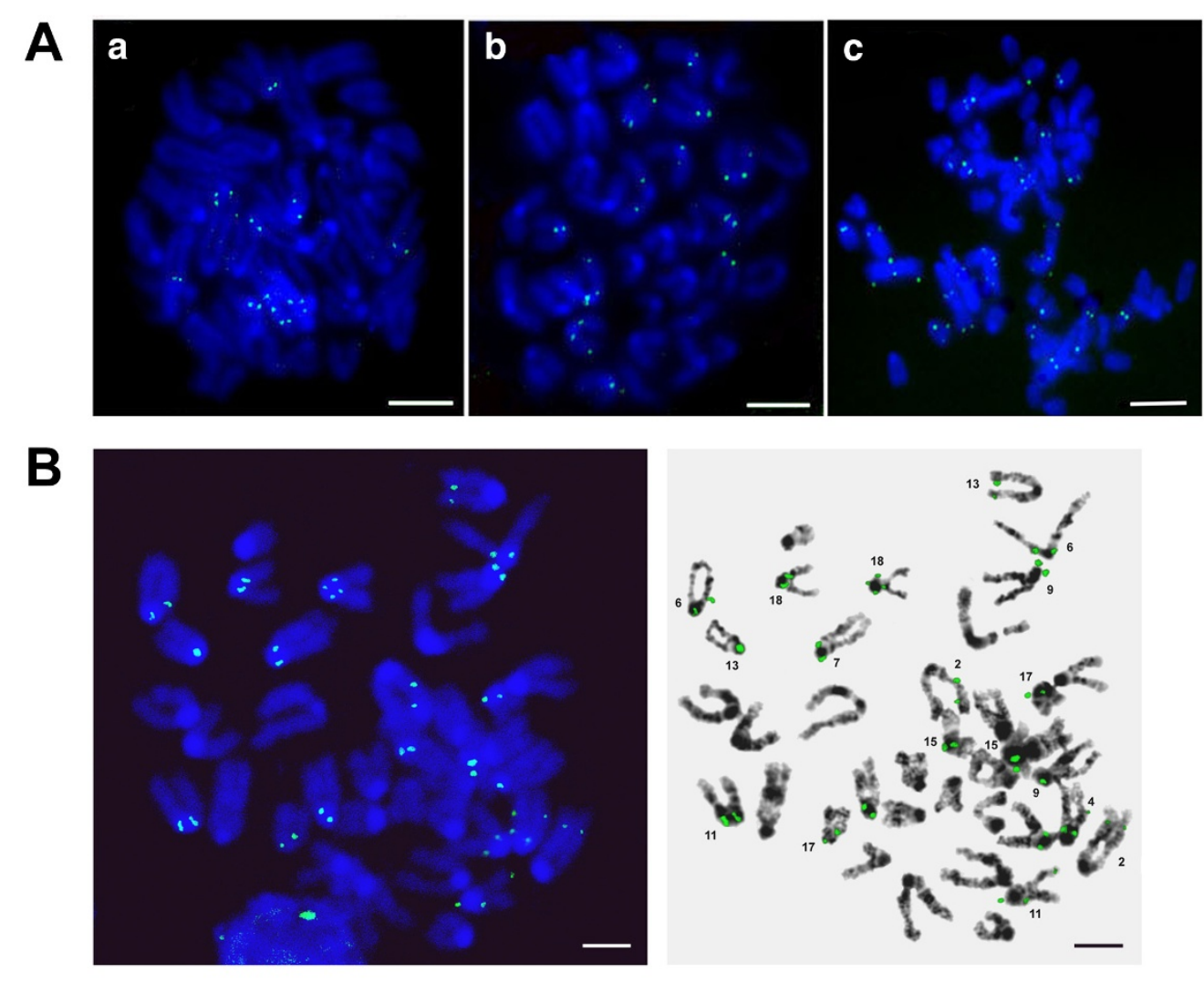

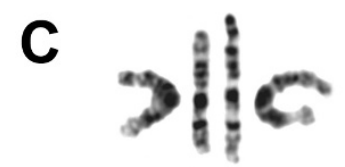

1

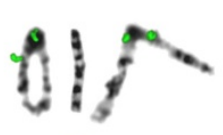

6

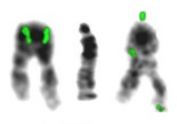

11

\section{at}

16

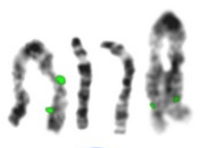

2

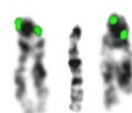

7

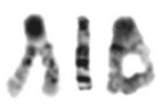

12

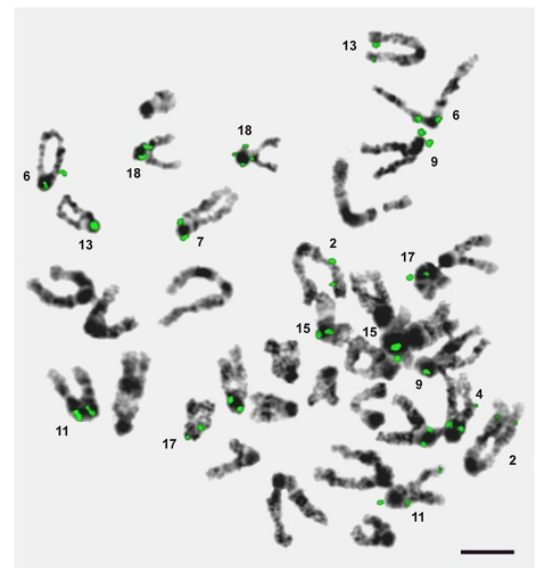

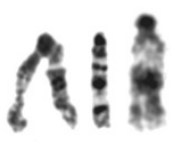

3

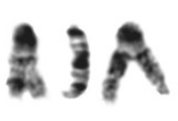

8

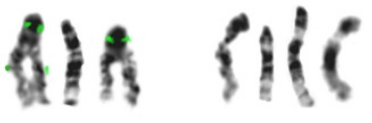

4

5

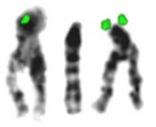

9

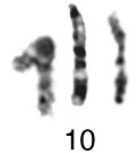

gil

13

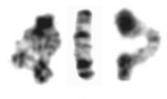

14

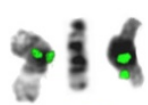

15

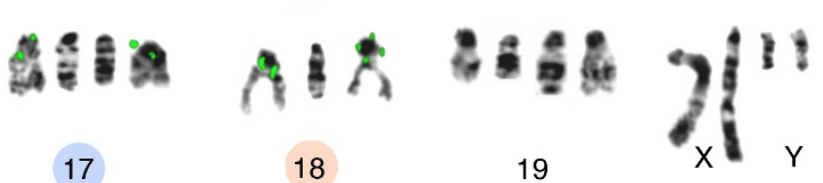

Figure 10 FISH with TR-22A-MM probe. A: Primary bone marrow metaphase plates ( $a$, b) and metaphase plate from cell line L929 (c). DAPI is blue, FISH signal is green; bar - $5 \mu \mathrm{m}$. B: one of the bone marrow metaphase plates with chromosome numbers indicated. Bar - $5 \mu \mathrm{m}$. C: in each chromosome group the middle image is G-banded mouse chromosome from atlas [41], the side (left and right) negative DAPI-banded chromosomes are from the plate shown on B. Ten chromosomes with the FISH signal are indicated by circles, four chromosomes with in situ signal that confirmed in silico prediction are indicated by orange circles. 


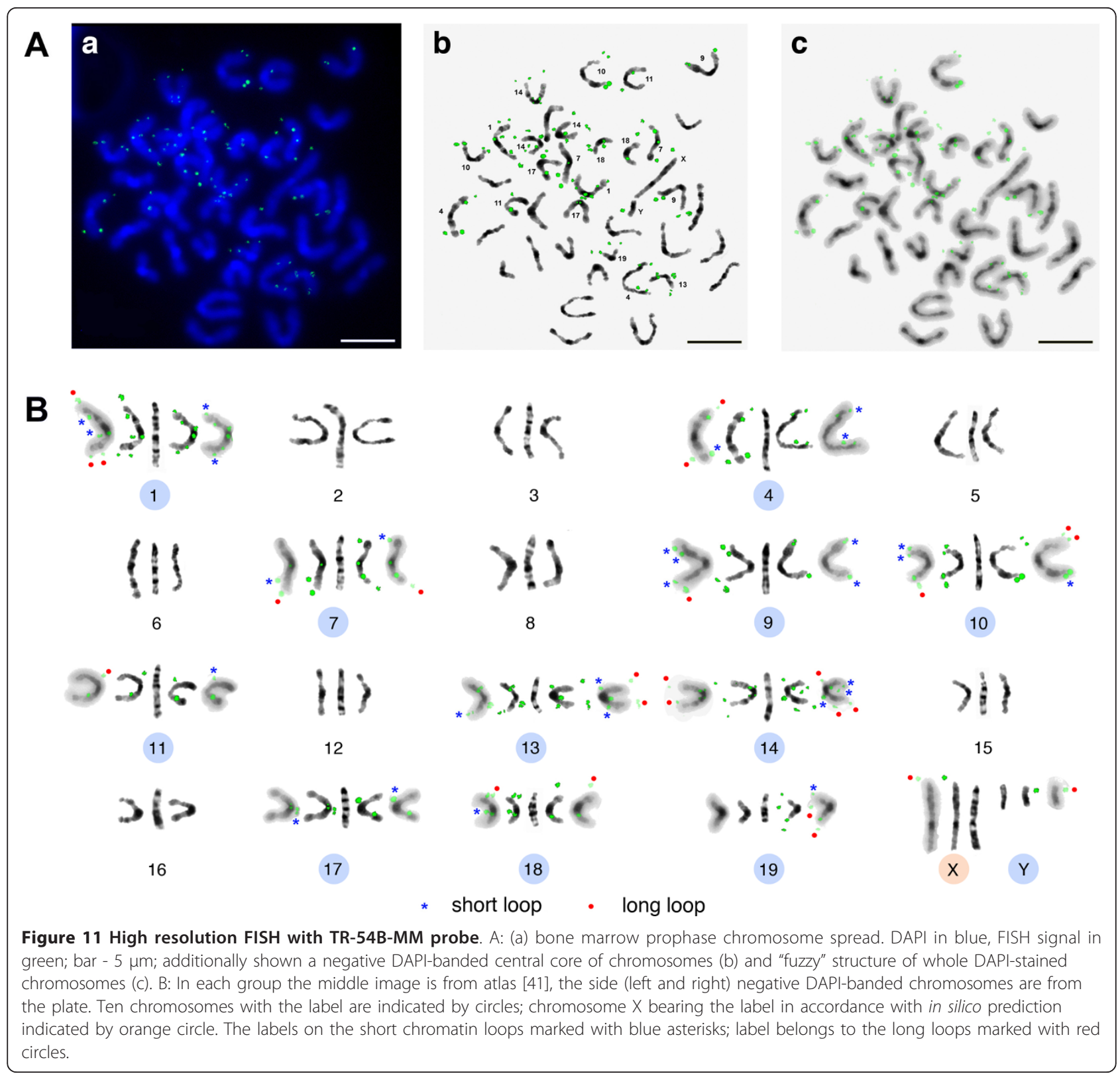

corresponded to chromosomes 1 and 14 [28]. The existence of a chromosome specific MiSat implies that the rate of sequence exchanges between non-homologous chromosomes relative to the rate of exchange between homologous chromosomes is much lower than was postulated previously. Based on these results the suggestion was made that the high degree of sequence homogeneity of both known mouse satDNA may reflect recent common ancestry [28]. Still, none of these probes have been worked up to be a reliable cytogenetic marker. Since only a few MiSat arrays were found in WGS (Additional file 1, Table S2), this does not give much hope to a successful design of a chromosome-specific probe with purely bioinformatics approach.

\section{Classical satellites}

Big classical satDNA are well known and were studied extensively. Human satDNA 1-4 (HS 1-4) are based on a "simple" 5-6 bp motif and HS3 is mostly well investigated $[10,11]$. HS3 was found in pericentromeric regions of all chromosomes but $2,6,8,11,12,18,19$, and $\mathrm{X}$ $[60,61]$. Chromosome-specific subfamilies of HS3 have been determined $[62,63]$ and those that belongs to chromosome 1 (HS3-1) and 9 (HS3-9) are two of them [61]. 
Mouse TRPC-21A resembles human "classical" or "simple sequence" satellites in most features. The dotplot at a high magnification suggests that units of $\sim 5-7$ bp could be distinguished inside the basic 21 bp monomer (grey lines between black ones on Figure $5 \mathrm{C}$ ), although the degree of diversity demands a special investigation to determine the exact oligonucleotide sequence. All of the TRPC-21A arrays contain HORs, it is common characteristic of classical satellites. Letters "PC" are included in the TRPC-21A name to indicate strictly pericentromeric location determined according to the relevant WGS position, position in the assembled genome (Tables 3 and 4) and confirmed by the FISH signal (Figures 8 and 9). The most prominent chromosome-specific probe was designed at the base of chromosome 3 variant of TRPC-21A (Table 4; Additional file 3, Figure S2; Additional file 1, Table S6).

TRPC-21A was the first tested, but among ML family some of the family members (TR-22A, TR-27A, and TR-31A) look promising for chromosomes-specific probes design due to HORs and their presence in the ChrUn. TR-22A and TR-27A were also found at the end of assembled chromosomes (Table 3), so the possibility to map them by FISH is quite high.

\section{GC content}

It is notable that most of newly found subfamilies have GC content higher than MaSat and MiSat - the mean for TRPC-21A is $~ 50 \%$, and even higher for ML family - $57 \%$ (Figure 2). Both GC-rich and AT-rich satDNA are known in human and most of the high eukaryotes $[64,65]$, hence our results cure the strange asymmetric satDNA distribution reported for mouse until now. The isochors (regions differ in GC content) have the functional significance for optimization of epigenetic genome regulation and supports the notion that noncoding DNA is important for orderly chromatin condensation and chromatin-mediated suppression of tissue-specific genes [66]. The absolute values of thermostability, bendability and ability to $\mathrm{B} \pm \mathrm{Z}$ transition correlated positively with the GC content, whereas curvature correlated negatively [67]. Although these conclusions were made on the base of introns and intergenic spacers as examples of noncoding DNA, TR of different GC and AT content may add to the isochoric genome structure due to its abundance in some regions.

\section{Bar code}

Alpha-satDNA is the only functional DNA sequence associated with all naturally occurring human centromeres. Two distinct forms of alpha-satellite are recognized based on their organization and sequence properties. A large fraction of alpha-satellite is arranged into HOR arrays where corresponding monomers are organized as multimeric repeat units ranging in size from 3 to $5 \mathrm{Mb}[68,69]$. Human chromosome-specific probes based on alpha-satDNA [70,71], "classical" satDNA [34], and megasatellites [6] exist and are used in cytogenetic analysis. It appears that using human WGS and assembled genome a set of TR characteristic for each human chromosome could be found, suggesting that TR might provide a kind of "bar code" for each chromosome.

The lack of mouse chromosome-specific probes causes problems for most genome-connected studies, including studies in developmental biology. Using WGS we have identified 62 subfamily of large tandemly repeated DNA. The next step is to map most of them to check whether there is the chromosome specificity in the hybridization pattern. Probably, it will be possible to create individual chromosome "bar-code" set of probes to be used in cytogenetic analysis. We suggest that this "bar-code" describes the heterochromatin signature for each chromosome and these signatures help to arrange chromosomes in the nucleus in the specific order during development. Potentially, this "bar-code" or signature represents the hypothetical Master Development Program, previously attributed to the heterochromatic regions [72].

\section{Conclusions}

Eight families including 62 subfamilies are found and characterized here by bioinformatics analysis. Most of them are more GC-rich than well known MaSat and MiSat. HOR structure was determined for some of them suggesting the existence of TR chromosome-specific variants. Probes for the representatives of three TR families were designed on the base of TR monomers units. In situ hybridization signal positions are in accordance with in silico predictions on the reference genome, although other chromosomes are labelled due to the poor assembly of the heterochromatic genome regions. A long probe based on chromosome 3 variant of TRPC-21A recognizes the longest fields of TR at the ends of chromosomes 3 and 17. No reliable cytogenetic probe was designed up to now. We suppose that with the future investigation of the newly characterized TR families it will be possible to determine the set of mouse chromosome-specific TR.

\section{Methods}

\section{Sequence databases}

Mouse sequences were obtained from NCBI ftp site in FASTA format: two WGS assemblies for projects AAHY and CAAA [73]; the reference genome assembly build 37.1 and Celera genome assembly build 37.1 [74]; MGSC genome assembly release 3 [75]. The genome banding annotation was obtained from the NCBI ftp site 
[76]. The Repbase database version 15.07 in FASTA format was obtained from [77]. To compile local blast databases we used blastdb program from BLAST+ suite with default parameters.

\section{Programs and search parameters used}

Sequence alignments were performed using blastn and bl2seq from BLAST+ suite [78]. Several search parameters were changed to work with repetitive DNA: max_target_seqs (the maximum number of database sequences for which any alignment will be reported) and num_descriptions (the maximum number of oneline descriptions of significant database sequenced reported) were set to 10,000, evalue (expectation value threshold for saving hit) was set to $10^{-16}$, word_size for word finder algorithm was set to 10, dust (arguments to DUST filtering algorithm) was set to "no", soft_masking parameter (simple repeat filter) was set to "false". All other search parameters were set to default values. Tandem repeat search was performed using TRF [23]. Search parameter mismatch was set to 5; maximum period size was set to 2000. Other search parameters were set to the default values. Self dot-plot matrix computations were done with in-house software with two sets of parameters: (1) window size set to $13 \mathrm{bp}$ and similarity indicated by gray-scale color from black (100\% window match) to white (100\% window mismatch); (2) window size set to $51 \mathrm{bp}$ and similarity indicated by two colors: black for $>90 \%$ (MaSat arrays) or $>80 \%$ (TRPC-21A arrays) window match; white for corresponding mismatch. To store the mouse large tandem repeat collection we used MySQL 5.1 database. TRF output analysis was performed with custom Python scripts. 3D-plots were rendered with Mathematica ${ }^{\mathrm{TM}}$ 7.0. A coordinate representation of mouse chromosome ideogram [76] and band position of DNA repeats were used for chromosome ideogram drawing with custom Python script.

\section{TR analysis}

To eliminate any redundant entries from the TRF output, all embedded TR arrays were discarded; in the case when two arrays had the same sequence coordinates a TR with a larger unit size was discarded. Overlapping arrays were considered as independent arrays. Repbase version 15.07 was used to compare TR with known repeats [79]. To remove false positive matches from Blast versus Repbase results, all matches that covered by repeats from Repbase less than $80 \%$ were discarded. Each pair of arrays was compared using bl2seq. We got a number of false-positive alignments due to the tandem nature of compared sequences. To remove false-positive or suspicious alignments we discarded all pair matches with a score less than 90. The remaining arrays were separated in subfamilies by Blast defined similarity. Two tandem repeats were placed in the same subfamily if they have a bl2seq match with score greater than 90 . Finally, each subfamily checked by hand for errors. In several cases subfamily exact borders are fuzzy (TR29A/B; TR-4A/B; TR-81A and TR-27A; TR-54A/B; TR$38 \mathrm{~A} / \mathrm{B})$. Those subfamilies pairs can be joined in one bigger subfamily with less strict Blast parameters.

\section{Mouse genome databases comparison}

We used three mouse genome assembles: the reference genome, the alternate (Celera) genome и MGSC genome assembly. Each genome has Chromosome Unknown (CrhUn) that contains all unplaced or unmapped contigs remained after assembly. TRF search with the parameters same as described has been applied to these 6 databases (Additional file 1, Table S7). There are prominent differences in total and large TR amount in different databases. It can be explained with a difference in the methodology of genome sequencing and assembly $[80,81]$. In the genome assembly process the additional sequence sources (e.g. clone based sequence) were used [21], which caused the difference in TR number found in WGS and genome assemblies. We used the reference genome build 37.1 as the most comprehensible, widely used and containing the largest amount of large TR to map newly found TR. The reference genome build 37.1 was assembled without WGS sequences and it has small ChrUn. Alternate (Celera) ChrUn was used to check the amount of TR found in WGS (Additional file 1, Table S7). We did not use MGSC genome and ChrUn assemblies as outdated with the lack of $\mathrm{Y}$ chromosome [21].

\section{Probe design}

The probes 1 and 4 tested in FISH the probe were designed as follows. Fragment composed of several monomers with a total length $\sim 150 \mathrm{bp}$ was chosen from the most variable region of tandem array, and it was flanked by two different adapters (Additional file 1, Table S6 and Additional file 3, Figure S2). The probe was amplified with primers to adapters and labelled with biotine-dUTP by PCR: $95^{\circ} \mathrm{C} 15 \mathrm{sec}, 60^{\circ} \mathrm{C} 30 \mathrm{sec}$, $72^{\circ} \mathrm{C} 30 \mathrm{sec}, 20$ circles. Probes 2 and 3 were synthesized as 3'-15'-biotine labelled (Beagle, St. Petersburg, Russia).

\section{Mitotic chromosomes}

Chromosome spreads from bone marrow cells were made according to the previously published method [82]. Colchicine $(0.4 \mathrm{ml} 0.04 \%$ solution) was injected intraperitoneally for $90 \mathrm{~min}$ before mice were sacrificed by cervical dislocation under anaesthesia. Bone marrow was washed out from legs tubular cylindrical bones with $75 \mathrm{mM} \mathrm{KCl}$. Suspension was incubated $15 \mathrm{~min}$ in $37^{\circ} \mathrm{C}$ and centrifuged $5 \mathrm{~min} 1000 \mathrm{rpm}$. Pellet was fixed 15 
min in cold fixative (methanol: acetic acid - 3:1) at $4^{\circ} \mathrm{C}$. Suspension and centrifugation cycles were repeated three times. At last, the suspension was dropped on wet cold slides, which were air dried to get rid of the fixative. Metaphase plates of good quality were selected under microscope.

L929 cells were cultured in Petri dishes in full medium (DMEM+10\% FCS) until 40-50\% confluency. The cells were treated with colcemid $(0.5 \mu \mathrm{g} / \mathrm{ml})$ for $2 \mathrm{~h}$ before harvesting. After treatment with hypotonic solution (50 mM KCl: $1 \% \mathrm{Na}$ citrate, 1: 1) for $20 \mathrm{~min}$ at $37^{\circ} \mathrm{C}$ the cells were fixed with acetic acid: methanol (1: $3)$, dropped onto ice-cold glass slides and air-dried. The slides were kept at $-20^{\circ} \mathrm{C}$ until FISH was performed.

\section{FISH with double stranded probes}

FISH with double stranded probes was done in the usual way [29]. The labeled probes were dissolved in the hybridization mixture $(50 \mu \mathrm{g} / \mu \mathrm{l}$ sheared yeast total DNA, 50\% formamid, 10\% dextran sulphate, 2x SSC), loaded on a slide with cells, covered with a smaller cover slip, and sealed with rubber cement. Slides were denatured simultaneously on a hot-block at $75^{\circ} \mathrm{C}$ for 2 min. Hybridization was performed overnight at $37^{\circ} \mathrm{C}$ in a humid chamber. Post-hybridization washes were done at $42^{\circ} \mathrm{C}$ in $50 \%$ formamid for $10 \mathrm{~min}$, twice in $2 \mathrm{x} \mathrm{SSC}$ for $5 \mathrm{~min}, 0.5 \mathrm{x}$ SSC for $10 \mathrm{~min}$ and finally in $2 \mathrm{xSSC}$ for $10 \mathrm{~min}$ at room temperature. The slides were counterstained with $0.5 \mu \mathrm{g} / \mathrm{ml}$ DAPI in $2 \mathrm{xSSC}$ solution for 5 min, rinsed in $2 x S S C$, and mounted in Citifluor antifade solution (Citifluor Ltd, UK).

\section{FISH with single stranded oligonucleotide probes}

FISH with single stranded probes was done according to a published protocol [83] with the following modifications. Oligonucleotides were synthesized as 3'-15'-biotine labeled for probes 2 and 3 (Additional file 1, Table S6). After RNase and pepsin pretreatment, metaphase chromosome spreads were dehydrated in ethanol series and air-dried. Chromosomes were denatured for $2 \mathrm{~min}$ in $70 \%$ formamide, 2 ü $\mathrm{SSC}$, at $65^{\circ} \mathrm{C}$. After being dehydrated in an ice-cold ethanol series of washes, hybridization was performed for $12-16 \mathrm{~h}$ at $37^{\circ} \mathrm{C}$. The hybridization solution contained $5 \mathrm{ng} / \mathrm{ml}$ probe, sheared yeast total DNA $(50 \mu \mathrm{g} / \mu \mathrm{l}), 25 \%$ formamide, $4 \times \mathrm{SSC}$. After hybridization, the slides were washed three times for $5 \mathrm{~min}$ in $2 \times \mathrm{SSC}$ at room temperature. For detection, preparations were incubated with fluorescein avidin D (Vector Laboratories) $(5 \mu \mathrm{g} / \mathrm{ml}$ in $2 \times$ SSC containing $5 \% \mathrm{BSA}$ ) for $40 \mathrm{~min}$ at room temperature. Then they were washed three times for $8 \mathrm{~min}$ in $2 \times \mathrm{SSC}$ at room temperature. Signal amplification was performed by treating the slides with a biotinylated goat anti-avidin
(Vector Laboratories) $(5 \mu \mathrm{g} / \mathrm{ml}$ in $2 \times$ SSC plus $5 \%$ BSA) for $40 \mathrm{~min}$ at room temperature. Preparations were washed again three times for $5 \mathrm{~min}$ with $2 \times \mathrm{SSC}$ and a new incubation with fluorescein avidin D (Vector Laboratories) were carried out for $40 \mathrm{~min}$ at room temperature. The slides were counterstained with $0.5 \mu \mathrm{g} / \mathrm{ml}$ DAPI in $2 \times$ SSC solution for $5 \mathrm{~min}$, rinsed in $2 \times \mathrm{SSC}$ and mounted in Citifluor antifade solution (Citifluor Ltd, UK).

\section{Microscopy and Image Acquisition}

For image acquisition the confocal microscope Leica TCS SP5 equipped with immersion $100 \times$ objective, 488 $\mathrm{nm}$ argon and $405 \mathrm{~nm}$ diode lasers was used. For primary image analysis Leica LAS AF software was used. The series of confocal sections were collected with the step size $0.25 \mu \mathrm{m}$, and maximal projections of the series were obtained. Negative (inverse) DAPI-banding pattern that is coincided with G-banding one was computer processed according to the protocol published [84]. Chromosome identification was going on with the help of images of individual G-banded mouse chromosomes with different level of compaction [41].

\section{Additional material}

Additional file 1: Supplementary tables. This file can be viewed with: Adobe Acrobat Reader.

Additional file 2: Coordinates of MaSat arrays. This file can be viewed with: Adobe Acrobat Reader.

Additional file 3: Supplementary figures. This file can be viewed with: Adobe Acrobat Reader.

\section{Acknowledgements}

The work was supported by MCB grant from the Russian Academy of Sciences, RFBR 11-04-01700-a and NIH/NCI R01 CA127378-01A1 grant. The authors thank Dr. A. Fedorov, Dr. A. Voronin and Dr. I. Kuznetsova for the fruitful discussions during the work. Viktor Nikonov is thanked for help with 3D-plot rendering in Wolfram Mathematica ${ }^{\mathrm{TM}}$ 7.0. Alena Yakusheva is thanked for the pictures design. We are grateful to Dr. D. Kipling, Dr. K. I. Galaktionov, and B. A. Dianova for helpful comments and language revision of the manuscript. We would like to thank two anonymous reviewers and Dr. M. Gelfand for careful reading and helpful comments, which improve manuscript.

\section{Author details}

${ }^{1}$ Institute of Cytology RAS, 4 Tikhoretsky avenue, 194064, St. Petersburg, Russia. ${ }^{2}$ Faculty of Biology and Soil Sciences, St. Petersburg State University, Universitetskaya nab. 7/9, St. Petersburg 199034, Russia. ${ }^{3}$ Department of Anatomy and Cell Biology, University of Florida College of Medicine, 1376 Mowry, Gainesville FL 32610-3633, USA.

\section{Authors' contributions}

GEV and DSJ performed probe labelling, FISH and chromosome identification. KAS performed genomic analysis and probe design. IAM participated in data interpretation and helped to draft the manuscript. POI and KAS conceived the experimental design, analyzed and interpreted data. $\mathrm{POI}$ wrote the final manuscript and coordinated the project. All authors have read and approved the final manuscript. 
Received: 5 February 2011 Accepted: 28 October 2011

Published: 28 October 2011

\section{References}

1. Morris CA, Moazed D: Centromere assembly and propagation. Cell 2007, 128:647-50.

2. Martienssen RA: Maintenance of heterochromatin by RNA interference of tandem repeats. Nature genetics 2003, 35:213-4.

3. Alleman M, Sidorenko L, McGinnis K, Seshadri V, Dorweiler JE, White J, Sikkink K, Chandler VL: An RNA-dependent RNA polymerase is required for paramutation in maize. Nature 2006, 442:295-8.

4. Enukashvily NI, Malashicheva AB, Waisertreiger IS-R: Satellite DNA spatial localization and transcriptional activity in mouse embryonic E-14 and IOUD2 stem cells. Cytogenetic and genome research 2009, 124:277-87.

5. Ames D, Murphy N, Helentjaris T, Sun N, Chandler V: Comparative analyses of human single- and multilocus tandem repeats. Genetics 2008, 179:1693-704.

6. Warburton PE, Hasson D, Guillem F, Lescale C, Jin X, Abrusan G: Analysis of the largest tandemly repeated DNA families in the human genome. BMC genomics 2008, 9:533.

7. Schueler MG, Higgins AW, Rudd MK, Gustashaw K, Willard HF: Genomic and genetic definition of a functional human centromere. Science 2001, 294:109-15.

8. Rudd MK, Willard HF: Analysis of the centromeric regions of the human genome assembly. Trends in genetics 2004, 20:529-33.

9. Choo KH: Centromere DNA dynamics: latent centromeres and neocentromere formation. American journal of human genetics 1997, 61:1225-33.

10. Lee C, Wevrick R, Fisher RB, Ferguson-Smith MA, Lin CC: Human centromeric DNAs. Human genetics 1997, 100:291-304.

11. Moyzis RK, Albright KL, Bartholdi MF, Cram LS, Deaven LL, Hildebrand $C E_{\text {, }}$ Joste NE, Longmire JL, Meyne J, Schwarzacher-Robinson T: Human chromosome-specific repetitive DNA sequences: novel markers for genetic analysis. Chromosoma 1987, 95:375-86.

12. Prosser J, Frommer M, Paul C, Vincent PC: Sequence relationships of three human satellite DNAs. Journal of molecular biology 1986, 187:145-55.

13. Podgornaya O, Dey R, Lobov I, Enukashvili N: Human satellite 3 (HS3) binding protein from the nuclear matrix: isolation and binding properties. Biochimica et biophysica acta 2000, 1497:204-14.

14. Kipling D, Ackford HE, Taylor BA, Cooke HJ: Mouse minor satellite DNA genetically maps to the centromere and is physically linked to the proximal telomere. Genomics 1991, 11:235-41.

15. Kalitsis P, Griffiths B, Choo KHA: Mouse telocentric sequences reveal a high rate of homogenization and possible role in Robertsonian translocation. Proc Natl Acad Sci 2006, 103:8786-91.

16. Wong AK, Rattner JB: Sequence organization and cytological localization of the minor satellite of mouse. Nucleic acids research 1988, 16:11645-61.

17. Guenatri M, Bailly D, Maison C, Almouzni G: Mouse centric and pericentric satellite repeats form distinct functional heterochromatin. The Journal of cell biology 2004, 166:493-505.

18. Broccoli D, Miller OJ, Miller DA: Relationship of mouse minor satellite DNA to centromere activity. Cytogenetics and cell genetics 1990, 54:182-6.

19. Hörz W, Altenburger W: Nucleotide sequence of mouse satellite DNA. Nucleic acids research 1981, 9:683-96.

20. Pertile MD, Graham AN, Choo KHA, Kalitsis P: Rapid evolution of mouse Y centromere repeat DNA belies recent sequence stability. Genome research 2009, 19:2202-13.

21. Waterston RH, Lindblad-Toh K, Birney E, et al: Initial sequencing and comparative analysis of the mouse genome. Nature 2002, 420:520-62.

22. Mural RJ, Adams MD, Myers EW, et al: A comparison of whole-genome shotgun-derived mouse chromosome 16 and the human genome. Science 2002, 296:1661-71.

23. Benson G: Tandem repeats finder: a program to analyze DNA sequences. Nucleic acids research 1999, 27:573-80.

24. Abdurashitov MA, Chernukhin VA, Gonchar DA, Degtyarev SK: Glal digestion of mouse gamma-satellite DNA: study of primary structure and ACGT sites methylation. BMC genomics 2009, 10:322.

25. Vissel B, Choo KH: Mouse major (gamma) satellite DNA is highly conserved and organized into extremely long tandem arrays: implications for recombination between nonhomologous chromosomes. Genomics 1989, 5:407-14.
26. Kidd JM, Sampas N, Antonacci F, Graves T, Fulton R, Hayden HS, Alkan C, Malig M, Ventura M, Giannuzzi G, Kallicki J, Anderson P, Tsalenko A, Yamada NA, Tsang P, Kaul R, Wilson RK, Bruhn L, Eichler EE: Characterization of missing human genome sequences and copynumber polymorphic insertions. Nature methods 2010, 7:365-71.

27. She X, Horvath JE, Jiang Z, Liu G, Furey TS, Christ L, Clark R, Graves T, Gulden CL, Alkan C, Bailey JA, Sahinalp C, Rocchi M, Haussler D, Wilson RK, Miller W, Schwartz S, Eichler EE: The structure and evolution of centromeric transition regions within the human genome. Nature 2004, 430:857-64.

28. Kipling D, Wilson HE, Mitchell AR, Taylor BA, Cooke HJ: Mouse centromere mapping using oligonucleotide probes that detect variants of the minor satellite. Chromosoma 1994, 103:46-55.

29. Kuznetsova IS, Enukashvily NI, Noniashvili EM, Shatrova AN, Aksenov ND, Zenin W, Dyban AP, Podgornaya Ol: Evidence for the existence of satellite DNA-containing connection between metaphase chromosomes. Journal of cellular biochemistry 2007, 101:1046-61.

30. Kuznetsova IS, Voronin AP, Podgornaya Ol: Telomere and TRF2/MTBP localization in respect to satellite DNA during the cell cycle of mouse cell line L929. Rejuvenation research 2006, 9:391-401.

31. Kuznetsova I, Podgornaya O, Ferguson-Smith M: High-resolution organization of mouse centromeric and pericentromeric DNA. Cytogenetic and genome research 2006, 112:248-55.

32. Broccoli D, Trevor KT, Miller OJ, Miller DA: Isolation of a variant family of mouse minor satellite DNA that hybridizes preferentially to chromosome 4. Genomics 1991, 10:68-74

33. Richard G-F, Kerrest A, Dujon B: Comparative genomics and molecular dynamics of DNA repeats in eukaryotes. Microbiol Mol Biol Rev 2008, 72:686-727.

34. Enukashvily NI, Donev R, Waisertreiger IS-R, Podgornaya Ol: Human chromosome 1 satellite 3 DNA is decondensed, demethylated and transcribed in senescent cells and in A431 epithelial carcinoma cells. Cytogenetic and genome research 2007, 118:42-54.

35. Namekawa SH, Payer B, Huynh KD, Jaenisch R, Lee JT: Two-step imprinted $\mathrm{X}$ inactivation: repeat versus genic silencing in the mouse. Molecular and cellular biology 2010, 30:3187-205.

36. Cooke HJ, Brown WR, Rappold GA: Hypervariable telomeric sequences from the human sex chromosomes are pseudoautosomal. Nature 1985, 317:687-92.

37. Cooke HJ, Brown WA, Rappold GA: Closely related sequences on human $X$ and $Y$ chromosomes outside the pairing region. Nature 1984, 311:259-61.

38. Giordano J, Ge Y, Gelfand Y, Abrusán G, Benson G, Warburton PE: Evolutionary history of mammalian transposons determined by genomewide defragmentation. PLoS computational biology 2007, 3:e137.

39. Smit AF: Identification of a new, abundant superfamily of mammalian LTR-transposons. Nucleic acids research 1993, 21:1863-72

40. Stocking C, Kozak CA: Murine endogenous retroviruses. Cellular and molecular life sciences 2008, 65:3383-98.

41. Mamaeva S: Atlas of chromosomes of human and animal cell lines Moscow: Scentific World; 2002, 1-236.

42. Ushiki T, Hoshi O: Atomic force microscopy for imaging human metaphase chromosomes. Chromosome Res 2008, 16:383-96.

43. Kireeva N, Lakonishok M, Kireev I, Hirano T, Belmont AS: Visualization of early chromosome condensation: a hierarchical folding, axial glue model of chromosome structure. The Journal of cell biology 2004, 166:775-85.

44. Alkan C, Cardone MF, Catacchio CR, Antonacci F, O'Brien SJ, Ryder OA, Purgato S, Zoli M, Della Valle G, Eichler EE, Ventura M: Genome-wide characterization of centromeric satellites from multiple mammalian genomes. Genome research 2010

45. Alkan C, Ventura M, Archidiacono N, Rocchi M, Sahinalp SC, Eichler EE: Organization and evolution of primate centromeric DNA from wholegenome shotgun sequence data. PLoS computational biology 2007, 3:1807-18

46. Mayer C, Leese F, Tollrian R: Genome-wide analysis of tandem repeats in Daphnia pulex - a comparative approach. BMC genomics 2010, 11:277.

47. Plohl M, Luchetti A, Mestrović N, Mantovani B: Satellite DNAs between selfishness and functionality: structure, genomics and evolution of tandem repeats in centromeric (hetero)chromatin. Gene 2008, 409:72-82. 
48. Podgornaya OI, Voronin AP, Enukashvily NI, Matveev IV, Lobov IB: Structurespecific DNA-binding proteins as the foundation for three-dimensional chromatin organization. International review of cytology 2003, 224:227-96.

49. Kobliakova I, Zatsepina O, Stefanova V, Polyakov V, Kireev I: The topology of early- and late-replicating chromatin in differentially decondensed chromosomes. Chromosome Res 2005, 13:169-81.

50. Yunis JJ, Yasmineh WG: Heterochromatin, satellite DNA, and cell function. Structural DNA of eucaryotes may support and protect genes and aid in speciation. Science 1971, 174:1200-9.

51. Grewal SIS, Elgin SCR: Transcription and RNA interference in the formation of heterochromatin. Nature 2007, 447:399-406.

52. Maison C, Bailly D, Peters AH, Quivy JP, Roche D, Taddei A, Lachner M Jenuwein T, Almouzni G: Higher-order structure in pericentric heterochromatin involves a distinct pattern of histone modification and an RNA component. Nature genetics 2002, 30:329-34.

53. Muchardt C, Guilleme M, Seeler JS, Trouche D, Dejean A, Yaniv M: Coordinated methyl and RNA binding is required for heterochromatin localization of mammalian HP1alpha. EMBO reports 2002, 3:975-81.

54. Lu J, Gilbert DM: Proliferation-dependent and cell cycle regulated transcription of mouse pericentric heterochromatin. The Journal of cell biology 2007, 179:411-21

55. Probst AV, Okamoto I, Casanova M, El Marjou F, Le Baccon P, Almouzni G: A strand-specific burst in transcription of pericentric satellites is required for chromocenter formation and early mouse development. Developmental cell 2010, 19:625-38.

56. Falconer E, Chavez EA, Henderson A, Poon SS, McKinney S, Brown L, Huntsman DG, Lansdorp PM: Identification of sister chromatids by DNA template strand sequences. Nature 2010, 463:93-7.

57. Biro PA, Carr-Brown A, Southern EM, Walker PM: Partial sequence analysis of mouse satellite DNA evidence for short range periodicities. Journal of molecular biology 1975, 94:71-86.

58. Southern EM: Long range periodicities in mouse satellite DNA. Journal of molecular biology 1975, 94:51-69.

59. Hayashi T, Ohtsuka H, Kuwabara K, Mafune Y, Miyashita N, Moriwaki K, Takahashi $Y$, Kominami R: A variant family of mouse minor satellite located on the centromeric region of chromosome 2. Genomics 1993, 17:490-2.

60. Gosden JR, Mtichell AR, Buckland RA, Clayton RP, Evans HJ: The location of four human satellite DNAs on human chromosomes. Cytogenetics and cell genetics 1975, 14:338-9.

61. Therkelsen AJ, Nielsen A, Kølvraa S: Localisation of the classical DNA satellites on human chromosomes as determined by primed in situ labelling (PRINS). Human genetics 1997, 100:322-6.

62. Higgins MJ, Wang HS, Shtromas I, Haliotis T, Roder JC, Holden JJ, White BN: Organization of a repetitive human $1.8 \mathrm{~kb} \mathrm{Kpnl}$ sequence localized in the heterochromatin of chromosome 15. Chromosoma 1985, 93:77-86.

63. Cooke HJ, Hindley J: Cloning of human satellite III DNA: different components are on different chromosomes. Nucleic acids research 1979, 6:3177-97.

64. Palomeque T, Lorite P: Satellite DNA in insects: a review. Heredity 2008, 100:564-73.

65. Beridze T: Satellite DNA Springer-Verlag; 1986, 149.

66. Vinogradov AE: Noncoding DNA, isochores and gene expression: nucleosome formation potential. Nucleic acids research 2005, 33:559-63.

67. Vinogradov AE: DNA helix: the importance of being GC-rich. Nucleic acids research 2003, 31:1838-44.

68. Mahtani MM, Willard HF: Pulsed-field gel analysis of alpha-satellite DNA at the human $\mathrm{X}$ chromosome centromere: high-frequency polymorphisms and array size estimate. Genomics 1990, 7:607-13.

69. Paar V, Pavin N, Rosandic M, Gluncic M, Basar I, Pezer R, Zinic SD: ColorHOR-novel graphical algorithm for fast scan of alpha satellite higher-order repeats and HOR annotation for GenBank sequence of human genome. Bioinformatics 2005, 21:846-852.

70. Warburton PE, Haaf T, Gosden J, Lawson D, Willard HF: Characterization of a chromosome-specific chimpanzee alpha satellite subset: evolutionary relationship to subsets on human chromosomes. Genomics 1996, 33:220-8.

71. Alexandrov I, Kazakov A, Tumeneva I, Shepelev V, Yurov Y: Alpha-satellite DNA of primates: old and new families. Chromosoma 2001, 110:253-266.

72. Parris GE: Scope of medical implications of the Master Development Program hypothesis. Medical hypotheses 2010, 74:953.
73. GenBank ftp site. [ftp://ftp.ncbi.nih.gov/genbank/wgs].

74. Mouse genome assembly build 37.1. [ftp://ftp.ncbi.nih.gov/genomes/ M_musculus/ARCHIVE/BUILD.37.1/Assembled_chromosomes/].

75. MGSC genome assembly release 3. [ftp://ftp.ncbi.nih.gov/genomes/ M_musculus/ARCHIVE/MGSCV3_Release3/Assembled_Chromosomes/].

76. Mouse ideograms. [ftp://ftp.ncbi.nih.gov/genomes/M_musculus/ARCHIVE/ BUILD.37.1/mapview/ideogram.gz].

77. Repbase collection version 15.7. [http://www.girinst.org/server/archive/ RepBase15.07/].

78. Camacho C, Coulouris G, Avagyan V, Ma N, Papadopoulos J, Bealer K, Madden TL: BLAST+: architecture and applications. BMC bioinformatics 2009, 10:421.

79. Jurka J, Kapitonov W, Pavlicek A, Klonowski P, Kohany O, Walichiewicz J: Repbase Update, a database of eukaryotic repetitive elements. Cytogenetic and genome research 2005, 110:462-7.

80. Blake JA, Bult CJ, Eppig JT, Kadin JA, Richardson JE: The Mouse Genome Database genotypes:::phenotypes. Nucleic acids research 2009, 37:D712-9.

81. Quinlan AR, Clark RA, Sokolova S, Leibowitz ML, Zhang Y, Hurles ME, Mell JC, Hall IM: Genome-wide mapping and assembly of structural variant breakpoints in the mouse genome. Genome research 2010.

82. Ford EH, Hamerton JL: A study of the mitotic chromosomes of mice if the strong a line. Exp Cell Res 1963, 32:320-6.

83. Tagarro I, Fernández-Peralta AM, González-Aguilera JJ: Chromosomal localization of human satellites 2 and 3 by a FISH method using oligonucleotides as probes. Human genetics 1994, 93:383-8.

84. Demin S, Pleskach N, Svetlova M, Solovjeva L: High-Resolution Mapping of Interstitial Telomeric Repeats in Syrian Hamster Metaphase Chromosomes. Cytogenetic and genome research 2011, 132:151-5.

doi:10.1186/1471-2164-12-531

Cite this article as: Komissarov et al:: Tandemly repeated DNA families in the mouse genome. BMC Genomics 2011 12:531.

\section{Submit your next manuscript to BioMed Central and take full advantage of:}

- Convenient online submission

- Thorough peer review

- No space constraints or color figure charges

- Immediate publication on acceptance

- Inclusion in PubMed, CAS, Scopus and Google Scholar

- Research which is freely available for redistribution

Submit your manuscript at www.biomedcentral.com/submit
C Biomed Central 